\title{
A three-dimensional cohesive-frictional grain-boundary micromechanical model for intergranular degradation and failure in polycrystalline materials
}

\author{
I. Benedetti ${ }^{\mathrm{a}, \mathrm{b}, 1}$, M.H. Aliabadi ${ }^{\mathrm{a}}$ \\ ${ }^{a}$ Department of Aeronautics, Imperial College London, South Kensington Campus, \\ SW7 2AZ, London, UK. \\ ${ }^{b}$ Dipartimento di Ingegneria Civile, Ambientale, Aerospaziale e dei Materiali, Università degli Studi di Palermo, Viale \\ delle Scienze, Edificio 8, 90128, Palermo, Italy.
}

\begin{abstract}
In this study, a novel three-dimensional micro-mechanical crystal-level model for the analysis of intergranular degradation and failure in polycrystalline materials is presented. The polycrystalline microstructures are generated as Voronoi tessellations, that are able to retain the main statistical features of polycrystalline aggregates. The formulation is based on a grain-boundary integral representation of the elastic problem for the aggregate crystals, that are modeled as threedimensional anisotropic elastic domains with random orientation in the three-dimensional space. The boundary integral representation involves only intergranular variables, namely interface displacement discontinuities and interface tractions, that play an important role in the micromechanics of polycrystals. The integrity of the aggregate is restored by enforcing suitable interface conditions, at the interface between adjacent grains. The onset and evolution of damage at the grain boundaries is modeled using an extrinsic non-potential irreversible cohesive linear law, able to address mixed-mode failure conditions. The derivation of the traction-separation law and its relation with potential-based laws is discussed. Upon interface failure, a non-linear frictional contact analysis is used, to address separation, sliding or sticking between micro-crack surfaces. To avoid a sudden transition between cohesive and contact laws, when interface failure happens under compressive loading conditions, the concept of cohesive-frictional law is introduced, to model the smooth onset of friction during the mode II decohesion process. The incrementaliterative algorithm for tracking the degradation and micro-cracking evolution is presented and discussed. Several numerical tests on pseudo- and fully three-dimensional polycrystalline microstructures have been performed. The influence of several intergranular parameters, such as cohesive strength, fracture toughness and friction, on the microcracking patterns and on the aggregate response of the polycrystals has been analyzed. The tests have demonstrated the capability of the formulation to track the nucleation, evolution and coalescence of multiple damage and cracks, under either tensile or compressive loads.
\end{abstract}

Keywords: Polycrystalline materials, Computational micromechanics, Intergranular failure, Cohesive-frictional laws, Boundary element method 


\section{Introduction}

The development of critical structures requires deep understanding of mechanisms governing degradation and failure of materials, especially when applications in severe environments are considered. Fracture modelling can be considered at different length scales: it is nowadays widely recognized that macroscopic material properties depend on the features of the microstructure. The knowledge of this link, which is the main subject of Micromechanics [1, 2, 3], is of relevant technological interest, as it may enable the design of materials with specific requirements in terms of strength, stiffness, ductility, toughness, etc., by means of suitable manipulations of the microstructural features that have a desired effect on the sought macroscopic property.

Polycrystalline materials, either metals, alloys or ceramics, are commonly employed in practical structures. Their microstructure, generally in the range from $10^{-6}$ to $10^{-3} \mathrm{~m}$, is characterized by features of the grains, their morphology, size distribution, anisotropy and crystallographic orientation, stiffness and toughness mismatch, and by physical and chemical properties of the intergranular interfaces [4]. All these aspects have a direct influence on the initiation and evolution of the microstructural damage, which is also sensitive to the presence of imperfections, flaws or porosity. Any theory or model for explaining failure mechanisms in this class of materials must then accommodate a relevant number of parameters [5].

Depending on a specific material and load and environment conditions, polycrystalline materials exhibit different failure mechanisms spanning from ductile to brittle fracture. Brittle failure, moreover, can be related to two main microstructural mechanisms, i.e. cleavage or transgranular fracture, in which the crack propagates on specific crystallographic planes within the grains, and intergranular failure, in which the microcracks follow the grain boundaries.

Brittle intergranular failure may occur in metals, alloys and ceramics [6] and it is particularly dependent on the chemical-physical state of the grains interfaces $[7,8,9]$, namely on the segregation of impurities and/or embrittling particles or on the presence of precipitate-free zones, in the case of precipitation-strengthened materials, $[10,11,12,13,14,15,16,17,18]$. Intergranular failure is also favored by hostile environmental conditions [19], by phenomena such as corrosion [20] or stress-corrosion cracking [21, 22] or by some combination of stress and high temperature [23]. The investigation and modelling of the brittle intergranular failure is then particularly relevant for better understanding and design with polycrystalline materials: in this framework it is worth noting that the concept of grain boundary engineering is now well established [24].

The microstructure of polycrystalline materials, and its influence on the microscopic failure mechanisms and on macroscopic material behavior, can be investigated by using different experimental techniques [25, 26, 27, 28, 29, 30, 31, 32, 33]. The experimental techniques for materials reconstruction and characterization are necessary for providing fundamental information and understanding; however, they require sophisticated equipment, careful material manufacturing and preparation, complicated postprocessing. They are generally expensive and time consuming. Such aspects are relevant whenever a truly three-dimensional (3D) characterization is pursued, or the simultaneous effect of several microstructural features is studied, or damage and failure at the microstructural level are investigated.

A viable alternative, or complement, to the experimental effort is offered by computational micromechanics. Several investigations have been devoted development of numerical models for

Email addresses: i.benedetti@imperial.ac.uk,ivano.benedetti@unipa.it (I. Benedetti), m.h.aliabadi@imperial.ac.uk (M.H. Aliabadi)

${ }^{1}$ Intra-European Marie Curie Fellow at Imperial College London, on leave from the University of Palermo, Italy. 
the analysis of polycrystalline microstructures and their failure processes, both in two and three dimensions [5]: among different approaches, multiscale methods appear to be the most promising, as they are able to bring together and link the diverse scales acting in the initiation and evolution of fracture $[34,35,36,37,38]$.

Several techniques have been developed and used for studying the crack initiation and propagation in heterogeneous or polycrystalline microstructures. Kamaya [39] employed the body force method for investigating 2D intergranular stress corrosion cracking. Sukumar et al. [40] studied the competition between inter- and transgranular crack growth in 2D polycrystalline brittle microstructure by using the extended FEM. Das et al. [41] coupled finite elements and cellular automata (CAFE) and showed the potential for microstructural and multiscale analysis of heterogeneous and polycrystalline materials. Also lattice models for quantitative estimates of the mechanical properties of polycrystalline microstructures and their damage have been reported [42]. The application and advantages of peridynamics for modelling crack initiation, propagation and fragmentation in polycrystalline ceramics are discussed in [36].

The above studies have mainly focused on the analysis of 2D polycrystalline problems, although some 3D applications of cellular automata and finite elements have been reported and the 3D potential of peridynamics has been discussed. There is currently an interest for development of truly 3D models for analysis of failure mechanisms in polycrystalline materials [5, 43, 44, 45]. This is motivated, on one hand, by the need for understanding complex inherently 3D phenomena (such as the influence of the geometry on the microcracking evolution; the competition between different failure modes, e.g. inter- and transgranular brittle propagation or the ductile-to-brittle transition; the grain-to-grain propagation of cleavage fracture [46]) and, on the other hand, by the need of complementing experimental investigations that, in the case of 3D microstructure reconstruction and characterization are particularly complex and expensive, especially when damage and failure are considered. Until recently, development of truly 3D models has been hindered by their excessive computational requirements. However, the present-day availability of cheaper and more powerful computational resources and facilities, namely high performance parallel computing, is favoring the advancement of the subject [47, 48, 49, 50].

A 3D rigid bodies-spring model for the analysis of brittle microcracking in polycrystalline Voronoi microstructures was developed by Toi and Kiyosue [51]. Marrow et al. [44] developed a simple 3D model for the analysis of intergranular stress corrosion cracking in austenitic stainless steel: in order to reduce the computational effort, in their model each grain is represented by a discrete system of elasto-plastic beams; grain boundaries are classified as either susceptible or resistant to intergranular fracture and different simplified failure criteria are assumed for them. Hughes et al. [45] developed a 3D geometrical model of the brittle fracture in polycrystalline zinc, with a focus on propagation of cleavage cracks from grain to grain. The stress corrosion intergranular crack initiation and growth in $3 \mathrm{D}$ polycrystalline microstructures was studied by using the finite element analysis by Kamaya and Itakura [52]. In their work the initiation and propagation of the cracks were modeled applying the concepts of damage mechanics. Musienko and Cailletaud [53] simulated inter- and transgranular stress corrosion cracking in polycrystalline aggregates with a finite element model in which the single grains exhibit viscoplastic behavior, transgranular cleavage is accommodated by introducing a pseudo-strain in the crystal plasticity framework and the grain boundaries are modeled as thin viscoplastic finite elements modified in the spirit of damage mechanics. Finite elements and continuum damage mechanics have been combined by Bomidi et al. for modelling of intergranular fatigue failure of 3D fine grain polycrystalline metallic MEMS devices [50].

A popular approach for modelling both $2 \mathrm{D}$ and $3 \mathrm{D}$ fracture problems in polycrystalline materials 
consists in the use of cohesive surfaces embedded in a finite element (FE) representation of the microstructure. The cohesive zone models provide a phenomenological framework in which the complex physical phenomena underlying the initiation and evolution of damage, in the so called process zone, are embedded in a traction-separation law expressing the progressive loss of material cohesion. In this way, initiation, propagation, branching and coalescence of microcracks stem as an outcome of the simulation, without any a priori assumptions. Several cohesive laws have been proposed [54, 55], but most popular are the potential-based laws by Tvergaard [56] and $\mathrm{Xu}$ and Needleman [57] and the linear laws by Camacho and Ortiz [58] and Ortiz and Pandolfi [59].

Cohesive FE models for 2D microstructures have been presented by several authors. Zhai and Zhou $[60,61]$ used the cohesive FE method for studying the quasi-static and dynamic failure in heterogeneous two-phase $\mathrm{Al}_{2} \mathrm{O}_{3} / \mathrm{TiB}_{2}$ ceramic microstructures. Espinosa and Zavattieri $[62,63]$ developed a grain level model for the analysis of intergranular failure initiation and evolution in brittle polycrystalline materials and discussed the features of different cohesive laws in numerical simulations. Wei and Anand [64] employed crystal plasticity theory and cohesive laws in a FE framework to study the dominant failure mechanisms in nanocrystalline fcc metals and carried out simulations on pseudo-3D microstructures (columnar grains). The dynamic fragmentation of granular ceramic microstructures has been studied with a cohesive FE scheme by Maiti et al. [65]. Zhou et al. [66] simulated crack growth in the polycrystalline microstructure of singlephase $\mathrm{Al}_{2} \mathrm{O}_{3}$ ceramic tool materials by introducing cohesive elements in the microstructure $\mathrm{FE}$ model. A 3D cohesive finite element model has been presented by Simonovski and Cizelj for intergranular cracks in a polycrystalline aggregate with elastic isotropic [48] and anisotropic, elastic and crystal plastic, grains [49].

An alternative to the FEM is the Boundary Element Method (BEM) that has proved effective for a variety of physical and engineering problems [67, 68]. A cohesive boundary element formulation for brittle intergranular failure in polycrystalline materials was proposed by Sfantos and Aliabadi [69], that subsequently used the developed technique in a multiscale analysis of polycrystalline material degradation and fracture [35, 37]. A 3D grain boundary formulation has recently been developed by Benedetti and Aliabadi for the material homogenization of polycrystalline materials [70].

In this work, a novel 3D grain-level model for the analysis of intergranular degradation and failure in polycrystalline materials is developed. The proposed formulation is given in Section2. The microstructure is generated using the Voronoi tessellations and the formulation is based on a grain-boundary integral representation of the elastic problem for the crystals, represented as anisotropic elastic domains with random orientation in space. The model is expressed in terms of interface displacement jumps and interface tractions, that play an important role in polycrystalline micromechanics. The continuity of the aggregate is retrieved by enforcing suitable conditions at the intergranular interfaces. The grain-boundary model given in Section3, which is the core of the present contribution, takes into account the onset and evolution of damage by means of an extrinsic irreversible linear cohesive law, able to address mixed-mode failure conditions. In order to distinguish energetically mode I from mode II failures, and make then the decohesion process energetically path-dependent, the requirement for existence of a cohesive potential is withdrawn and the form of the traction-separation law is directly given, departing from the potential-based derivation. Its relationship with potential laws used by several authors is however discussed in Section 3.3.2. Upon interface failure, a non-linear frictional contact analysis is introduced for addressing contact, either slip or stick, and separation between micro-crack surfaces. Moreover, in case the of interface mode II failure under compressive loading conditions, to avoid 
some non-physical behavior induced by the switch-off transition between cohesive and contact laws, the concept of cohesive-frictional law is introduced in Section 3.3.4. The incremental-iterative algorithm for tracking the micro- degradation and cracking evolution is presented and discussed in Section 4 and the advantages provided by the use of interface fields only are highlighted. Several numerical tests on pseudo- and fully-3D polycrystalline aggregates have been performed in Section 5, either in the case of tensile or compressive load. Directions of further research are eventually discussed in Section 6.

To the authors' knowledge, it is the first time that a comprehensive 3D boundary element model of the polycrystalline microstructure, with interface cohesive-frictional evolution, has been presented.

\section{Polycrystalline microstructure model}

In this section, the computational model for the analysis of polycrystalline microstructures is described.

\subsection{Artificial microstructure generation}

Artificial polycrystalline microstructures can be generated by using either experimental techniques or computer models able to render the main statistical features of the aggregate. Various experimental techniques are available for microstructural characterization $[25,26,27,28$, $29,71,72,73]$ and their output is sometimes used as input for finite element (FE) analysis $[74,75,48,49]$. However a complete 3D reconstruction still remains a challenging task. Moreover, experimental microstructures are often sampled in terms of voxels, and careful data processing is needed to avoid the introduction of model artifacts, for example stepped grain boundaries, that are not suitable for micro-cracking analysis. On the other hand, the use of reliable computer models offers the opportunity of analyzing large numbers of microstructures, complementing the experimental effort $[76,5]$ : synthetic microstructures and unstructured meshes, reflecting the actual micro-morphology, are powerful tools for the study of complex phenomena like material degeneration and fracture at the micro-scale.

In the case of polycrystals, a reliable computer representation must retain the main topological, morphological and crystallographic features of the microstructural aggregate. The Voronoi tessellations have been consistently adopted for representing polycrystalline materials at the grain scale $[77,78,79]$. Although it has been shown that they slightly misestimate some polycrystalline microstructural distributions $[25,80]$, they have however been widely successfully employed for modelling purposes $[5,81,80,82,83,84,85,86,70]$.

In any case, the Voroni tessellations have the advantage of being analytically defined and simple to generate. In this work, the free open-source C++ software library Voro++ (http:// math.1bl.gov/ voro++/; [87]) is used for generating the tessellation, Fig.(1). The representation is completed by assigning random or specific orientation to each crystal in the group $S O(3)$ of rotations in the three-dimensional space. For further details about the microstructure generation, the reader is referred to [70].

It is useful to give some details about the topology and morphology of the Voronoi tessellations and introduce some notation that will be used in the following. Each Voronoi cell $\mathscr{G}_{k}$ is a convex polyhedron bounded by the surface $\mathscr{B}^{k}$ and it represents a single crystal: it will be equivalently 
referred to as grain, cell or crystal. In general two different kinds of grains can be distinguished: the domain boundary grains, which intersect the external boundary $\mathscr{B}$ of the analysis region $\mathscr{C}$, and the internal grains, that are completely surrounded by other grains. A part $\mathscr{B}_{n c}^{k}$ of the surface of a domain boundary grain $\mathscr{G}_{k}$ lies on $\mathscr{B}$, while the remaining part $\mathscr{B}_{c}^{k}$ is the area of contact with the surrounding contiguous grains:

$$
\mathscr{B}^{k}=\mathscr{B}_{n c}^{k} \cup \mathscr{B}_{c}^{k} \quad \mathscr{B}_{n c}^{k}=\mathscr{G}_{k} \cap \mathscr{B} \quad \mathscr{B}_{c}^{k}=\mathscr{B}^{k}-\mathscr{B}_{n c}^{k}
$$

where the subscripts $n c$ and $c$ stand for non contact and contact, respectively. Of course $\mathscr{B}_{n c}^{k}$ only exists for the domain boundary grains, while for the internal grains $\mathscr{B}^{k}=\mathscr{B}_{c}^{k}$.

The grain surface $\mathscr{B}^{k}$ is given by the union of flat convex polygonal faces $\mathscr{F}_{j}^{k}$

$$
\mathscr{B}^{k}=\partial \mathscr{G}_{k}=\bigcup_{j=1}^{N_{f}^{k}} \mathscr{F}_{j}^{k}
$$

where $N_{f}^{k}$ is the number of flat faces of the $k-t h$ grain. Two contiguous faces share a straight cell edge while two contiguous edges meet at a cell vertex. It is worth noting that flat faces and straight edges are particularly suitable for numerical treatment. Each face $\mathscr{F}_{j}^{k}$ is associated with a unique outward unit normal vector $\mathbf{n}_{j}^{k}=\left\{n_{i}\right\}$. A local reference system $\left\{\tilde{x}_{1} \tilde{x}_{2} \tilde{x}_{3}\right\}_{j}^{k}$ is set on each grain face, so to express the local components of displacements and tractions of points belonging to that face of that grain. The local reference system on each face is chosen so that $\tilde{x}_{3} \equiv \mathbf{n}_{j}^{k}$ and the plane $\tilde{x}_{1} \tilde{x}_{2}$ contains the considered face, Fig.(2). Local components of displacements and tractions are denoted with a tilde, so that $\tilde{\mathbf{u}}_{j}^{k}$ and $\tilde{\mathbf{t}}_{j}^{k}$ refer to displacements and tractions of points belonging to the face $\mathscr{F}_{j}^{k}$, expressed in the local reference system associated with that face.

Two adjacent Voronoi cells, say $\mathscr{G}_{a}$ and $\mathscr{G}_{b}$, share a tessellation interface $\mathscr{I}_{a b}=\mathscr{G}_{a} \cap \mathscr{G}_{b}$. Let us assume that the $n-t h$ face $\mathscr{F}_{n}^{a}$ of $\mathscr{G}_{a}$ and the $m-t h$ face $\mathscr{F}_{m}^{b}$ of $\mathscr{G}_{b}$ are in contact. Then, if in the grain global numbering $a<b$, the local reference system $\left\{\tilde{x}_{1} \tilde{x}_{2} \tilde{x}_{3}\right\}_{n}^{a}$ on the face of $\mathscr{G}_{a}$ induces the local reference system $\left\{\tilde{x}_{1} \tilde{x}_{2} \tilde{x}_{3}\right\}_{m}^{b}$ on the contiguous face of $\mathscr{G}_{b}$, so that $\tilde{x}_{i}^{b} \equiv-\tilde{x}_{i}^{a}$ for $i=1,2,3$, Fig.(3). The introduction of these grain-face local reference systems simplify the writing of the interface equations, as it will be seen in Section 3.

\subsection{Anisotropic grain boundary element formulation}

In the present work, each crystal is modelled as a single elastic domain $\mathscr{G}_{k}$ with orthotropic linearly elastic behavior and random spatial orientation. The numerical model for the single crystal is obtained by using the Boundary Element Method (BEM) for three-dimensional anisotropic elasticity [88]. The polycrystalline aggregate is modelled as a multi-region boundary element problem, so that different elastic properties and spatial orientation can be assigned to each grain [68].

The constitutive equations for the grain material are

$$
\sigma_{i j}=c_{i j k l} \varepsilon_{k l}
$$

where the constants $c_{i j k l}$ denote the components of the stiffness tensor linking the components $\sigma_{i j}$ of the stress tensor to the components $\varepsilon_{k l}$ of the strain tensor. If the compact Voigt notation is used to denote the elements of the elastic tensors, see for example [89], the stiffness tensor can 
be written as a $(6 \times 6)$ matrix $\mathbf{C}=\left[C_{i j}\right]$, where $C_{11}=c_{1111}, C_{12}=c_{1122}, C_{16}=c_{1112}, C_{44}=c_{2323}$, etc. For the general orthotropic case, 9 constants are needed for the complete material characterization, due to material symmetries. The hypothesis of orthotropic material is not restrictive, as the majority of single metallic and ceramic crystals present general orthotropic behavior.

If a generic grain $\mathscr{G}_{k}$ with its own crystallographic orientation and an accordingly defined coordinate system is considered, the displacement boundary integral representation is given by [68]

$$
c_{i j}^{k}(\boldsymbol{y}) u_{j}^{k}(\boldsymbol{y})+\int_{\mathscr{B}_{n c}^{k} \cup \mathscr{B}_{c}^{k}} T_{i j}^{k}(\boldsymbol{x}, \boldsymbol{y}) u_{j}^{k}(\boldsymbol{x}) d \mathscr{B}(\boldsymbol{x})=\int_{\mathscr{B}_{n c}^{k} \cup \mathscr{B}_{c}^{k}} U_{i j}^{k}(\boldsymbol{x}, \boldsymbol{y}) t_{j}^{k}(\boldsymbol{x}) d \mathscr{B}(\boldsymbol{x})
$$

where $U_{i j}^{k}(\boldsymbol{x}, \boldsymbol{y})$ and $T_{i j}^{k}(\boldsymbol{x}, \boldsymbol{y})$ denote the displacement and traction fundamental solutions of the 3D anisotropic problem, see Appendix A, while $c_{i j}^{k}$ are the so called free terms, stemming from the boundary integral limiting procedure [68]. In Eq.(4), the displacement and traction components are expressed with respect to a unique reference system coincident with the axes of material orthotropy of each grain: this is because, when a reference system is not coincident with the material axes, an orthotropic grain will have a general anisotropic behavior. However, although it might slightly simplify the numerical computation of the fundamental solutions, this choice is not mandatory in the present work, since the fundamental solutions are computed using a scheme for general three-dimensional anisotropy [90].

The surface integrals in Eq.(4) are defined over the entire boundary $\mathscr{B}^{k}$ of the grain. According to Eq.(1), $\mathscr{B}_{n c}^{k}$ belongs to the external boundary of a microstructural cell and a suitable set of boundary conditions (BCs) must then be prescribed over it. In general, for a point $x \in \mathscr{B}_{n c}^{k}$ either displacement components $u_{i}^{k}(\boldsymbol{x})$ or the homologous traction components $t_{i}^{k}(\boldsymbol{x})$ are unknown, being the other quantity prescribed by BCs. On the contrary, on the contact surface $\mathscr{B}_{c}^{k}$, both displacements and tractions are unknowns. In order to solve the microstructural boundary value problem, Eqs.(4), written for each grain of the considered aggregate, must be complemented with an adequate set of boundary conditions and interface equations, relating displacements and tractions on the contiguous faces of adjacent crystals.

To simplify the expression of boundary conditions and interface equations, Eq.(4) is rewritten representing all the displacements and tractions in the local reference systems $\left\{\tilde{x}_{1} \tilde{x}_{2} \tilde{x}_{3}\right\}_{j}^{k}$ set over each face $\mathscr{F}_{j}^{k}$ of the grain, according to what explained in Section 2.1. The displacement boundary integral equations can be rewritten as

$$
\tilde{c}_{i j}^{k}(\boldsymbol{y}) \tilde{u}_{j}^{k}(\boldsymbol{y})+\sum_{q=1}^{N_{f}^{k}} \int_{\mathscr{F}_{q}^{k}} \tilde{T}_{i j}^{k}(\boldsymbol{x}, \boldsymbol{y}) \tilde{u}_{j}^{k}(\boldsymbol{x}) d \mathscr{F}(\boldsymbol{x})=\sum_{q=1}^{N_{f}^{k}} \int_{\mathscr{F}_{q}^{k}} \tilde{U}_{i j}^{k}(\boldsymbol{x}, \boldsymbol{y}) \tilde{t}_{j}^{k}(\boldsymbol{x}) d \mathscr{F}(\boldsymbol{x})
$$

where the tilde indicates local components and

$$
\tilde{c}_{i j}^{k}=c_{i n}^{k} R_{n j}^{q k} \quad \tilde{U}_{i j}^{k}=U_{i n}^{k} R_{n j}^{q k} \quad \tilde{T}_{i j}^{k}=T_{i n}^{k} R_{n j}^{q k}
$$

with the components $R_{i j}^{q k}$ defining the coordinate transformation linking the components of vector fields written in the material system and in the local system attached to the face $\mathscr{F}_{q}^{k}$ according to

$$
u_{i}^{k}=R_{i j}^{q k} \tilde{u}_{j}^{k} \quad t_{i}^{k}=R_{i j}^{q k} \tilde{t}_{j}^{k} .
$$

The boundary conditions can now be written as

$$
\tilde{u}_{i}^{k}(\boldsymbol{x})=\bar{u}_{i}^{k}(\boldsymbol{x}) \quad \text { or } \quad \tilde{t}_{i}^{k}(\boldsymbol{x})=\bar{t}_{i}^{k}(\boldsymbol{x})_{7} \quad i=1,2,3 \quad \forall \boldsymbol{x} \in \mathscr{F}_{j}^{k} \subset \mathscr{B}_{n c}^{k}
$$


where the $\bar{x}$ denotes a prescribed value for $x$. On the other hand, the interface equations can be expressed as

$$
\left\{\begin{array}{rl}
\Psi_{i}^{a b}\left[\tilde{u}_{j}^{a}(\boldsymbol{x}), \tilde{u}_{j}^{b}(\boldsymbol{x}), \tilde{t}_{j}^{a}(\boldsymbol{x}), \tilde{t}_{j}^{b}(\boldsymbol{x})\right] & =\psi_{i}^{a b} \\
\Phi_{i}^{a b}\left[\tilde{t}_{j}^{a}(\boldsymbol{x}), \tilde{t}_{j}^{b}(\boldsymbol{x})\right] & =0
\end{array} \quad i, j=1,2,3 \quad \forall \boldsymbol{x} \in \mathscr{I}_{a b},\right.
$$

on the interface between the grains $\mathscr{G}_{a}$ and $\mathscr{G}_{b}$. The equations $\Phi_{i}^{a b}$ can be generally interpreted as equilibrium equations while the terms $\Psi_{i}^{a b}$, depending on the evolution and internal state of the microstructure, may represent either continuity, cohesive or frictional contact laws, as it will be explained in Section 3. It is worth stressing that the interface equations involve local components of displacements and tractions of different grains.

To solve numerically the polycrystalline problem, each grain face $\mathscr{F}_{q}^{k}$ is discretized into a collection of non-overlapping triangular elements, Appendix B, and Eq.(5) is treated according to the classical boundary element collocation procedure [68], leading to the following set of equations for the single grain

$$
\left[\begin{array}{ll}
\tilde{\mathbf{H}}_{n c}^{k} & \tilde{\mathbf{H}}_{c}^{k}
\end{array}\right]\left\{\begin{array}{l}
\tilde{\mathbf{u}}_{n c}^{k} \\
\tilde{\mathbf{u}}_{c}^{k}
\end{array}\right\}=\left[\begin{array}{ll}
\tilde{\mathbf{G}}_{n c}^{k} & \tilde{\mathbf{G}}_{c}^{k}
\end{array}\right]\left\{\begin{array}{c}
\tilde{\mathbf{t}}_{n c}^{k} \\
\tilde{\mathbf{t}}_{c}^{k}
\end{array}\right\} .
$$

The system of equations for the entire polycrystalline aggregate is obtained by evaluating Eq.(10) for each grain and enforcing the boundary and interface conditions on the overall aggregate. The final system is given by

$$
\left[\begin{array}{ccccc}
\mathbf{A}^{1} & \mathbf{B}^{1} & \mathbf{0} & \cdots & \mathbf{0} \\
\mathbf{0} & \mathbf{A}^{2} & \mathbf{B}^{2} & \cdots & \mathbf{0} \\
\vdots & \vdots & \ddots & \vdots \\
\mathbf{0} & \cdots & \mathbf{0} & \mathbf{A}^{N_{g}} & \mathbf{B}^{N_{g}} \\
& \longleftarrow \text { Interface e equations } \mathbf{\Psi} \longrightarrow \\
\longleftarrow & \text { Interface equations } \mathbf{\Phi} \longrightarrow
\end{array}\right]\left\{\begin{array}{c}
\mathbf{x}_{n c}^{1} \\
\mathbf{x}_{c}^{1} \\
\vdots \\
\mathbf{x}_{n c}^{N_{g}} \\
\mathbf{x}_{c}^{N_{g}}
\end{array}\right\}=\left\{\begin{array}{c}
\mathbf{C}^{1} \mathbf{y}_{n c}^{1} \\
\mathbf{C}^{2} \mathbf{y}_{n c}^{2} \\
\vdots \\
\mathbf{C}^{N_{g}} \mathbf{y}_{n c}^{N_{g}} \\
\psi \\
\mathbf{0}
\end{array}\right\}
$$

where the matrix blocks $\mathbf{A}^{k}$ contain columns from the matrices $\tilde{\mathbf{H}}_{n c}^{k}$ and $-\tilde{\mathbf{G}}_{n c}^{k}$ corresponding to the unknown components of $\tilde{\mathbf{u}}_{n c}^{k}$ and $\tilde{\mathbf{t}}_{n c}^{k}$ that are collected in $\mathbf{x}_{n c}^{k}$, the blocks $\mathbf{C}^{k}$ collect columns from $-\tilde{\mathbf{H}}_{n c}^{k}$ and $\tilde{\mathbf{G}}_{n c}^{k}$ corresponding to the known components of $\tilde{\mathbf{u}}_{n c}^{k}$ and $\tilde{\mathbf{t}}_{n c}^{k}$, i.e. the BCs, that are collected in $\mathbf{y}_{n c}^{k}, \mathbf{B}^{k}=\left[\tilde{\mathbf{H}}_{c}^{k}-\tilde{\mathbf{G}}_{c}^{k}\right]$, the vectors $\mathbf{x}_{c}^{k}$ collect the unknown interface displacements and tractions of the $k$ - th grain and the matrices $\boldsymbol{\Psi}_{\text {and }} \boldsymbol{\Phi}$ implement the interface conditions (9) in the system matrix. It is worth noting that, in writing system (11), it has been implicitly assumed that the interface conditions can be expressed in matrix-vector product form. In general, this is not strictly true. Indeed, as already mentioned, the interface equations $\Psi_{i}^{a b}$ depend on the internal state $\mathscr{H}$ of the microstructure and it might not be possible to express them as linear functions of the interface displacements and tractions. However, in the present work, an incremental-iterative solution strategy is adopted and, during each iteration, a linear system of form (11) can be written and solved, as it will be detailed in Section 4.1.

System (11) can be rewritten in the more compact form as

$$
\left[\begin{array}{c}
\mathbf{M}(\mathrm{BCs}) \\
\mathbf{\Psi}(\mathscr{H}) \\
\mathbf{\Phi}
\end{array}\right] \mathbf{X}=\left\{\begin{array}{c}
\mathbf{y}(\mathrm{BCs}, \lambda) \\
\boldsymbol{\mathbf { 0 }}
\end{array}\right\} \quad \text { or } \quad \mathbf{A}(\mathscr{H}) \mathbf{X}=\mathbf{Y}(\lambda)
$$

where the dependency of the various matrix and vector blocks on the boundary conditions (BCs), on the internal state $\mathscr{H}$ of the microstructure and on the load factor $\lambda$ has been highlighted. 
The population of the blocks $\mathbf{A}^{k}$ and $\mathbf{B}^{k}$, appearing in system (11), is numerically demanding, due to need of integrating the anisotropic kernels in Eqs. (5). The use of efficient schemes can accelerate this stage: for further details, the reader is referred to [90], or to [91] for a more recent overview.

For microstructures with a high number of grains, the problem is suitable for parallel computing, as the matrices for different grains can be independently computed on different processors.

\section{Grain-boundary model}

The generic interface $\mathscr{I}$ between two grains, in polycrystalline materials, is subjected to the development and evolution of damage and cracks. Before proceeding with the description of the interface model, it is useful to give some notation and clarify the concept of interface pair.

\subsection{Interface description}

Let us assume that the $n-t h$ face $\mathscr{F}_{n}^{a}$ of $\mathscr{G}_{a}$ and the $m-t h$ face $\mathscr{F}_{m}^{b}$ of $\mathscr{G}_{b}$ meet at the interface $\mathscr{I}_{a b}$. Let us now assume that the interface $\mathscr{I}_{a b}$ is in the pristine state, so that $\mathscr{F}_{n}^{a} \equiv \mathscr{F}_{m}^{b} \equiv \mathscr{I}_{a b}$. This implies that the generic point $P \in \mathscr{F}_{n}^{a} \subset \mathscr{G}_{a}$ is perfectly bonded to the point $Q \in \mathscr{F}_{m}^{b} \subset \mathscr{G}_{b}$ and continuity of displacements and equilibrium of tractions hold. When the interface starts developing damage, the previous considerations are no longer valid and, in general, $\mathscr{F}_{n}^{a} \neq \mathscr{F}_{m}^{b}$, i.e. relative displacements between the two grain surfaces are now allowed. The two points $P$ and $Q$, that were coincident at the initial state, form an interface pair $\mathscr{P}$. If $u_{i}^{a}(P)$ and $u_{i}^{b}(Q)$ are the displacements associated to such points, in general $u_{i}^{a}(P) \neq u_{i}^{b}(Q)$ and the difference between them defines a displacement jump that, in local coordinates, can be expressed as

$$
\delta \tilde{u}_{i}^{a b}=-\left[\tilde{u}_{i}^{a}(P)+\tilde{u}_{i}^{b}(Q)\right] .
$$

The above definition is a consequence of the choice of the local reference systems, that are opposite on the two grain faces in contact. In particular, it is to be noted that the third component of the displacement jump

$$
\delta \tilde{u}_{3}^{a b}=-\left[\tilde{u}_{3}^{a}(P)+\tilde{u}_{3}^{b}(Q)\right]=\delta u_{n}^{a b}
$$

expresses the opening between the two surfaces and, according to the given definition, only $\delta \tilde{u}_{n}^{a b} \geq 0$ is allowed, while $\delta \tilde{u}_{n}^{a b}<0$ implies a violation of the impenetrability of grains.

On the other hand, if $\tilde{t}_{i}^{a}(P)$ and $\tilde{t}_{i}^{b}(Q)$ are the tractions associated to the two points, acting respectively on the surfaces $\mathscr{F}_{n}^{a}$ and $\mathscr{F}_{m}^{b}$ with normal vectors $n_{i}^{a}(P)$ and $n_{i}^{b}(Q)=-n_{i}^{a}(P)$, the traction equilibrium, that holds also when damage or cracks have developed, always requires that $\tilde{t}_{i}^{a}(P)=\tilde{t}_{i}^{b}(Q)$. The tractions $\tilde{t}_{i}^{a}(P)$ or $\tilde{t}_{i}^{b}(Q)$ can then be interchangeably used to express the traction state of the given pair.

Summarizing, the two points $P$ and $Q$, initially in contact, constitute an interface pair $\mathscr{P}$, whose state, during the microstructure evolution, is characterized by the level of damage, the displacement jump $\delta \tilde{u}_{i}^{a b}$ and the interface tractions $\tilde{t}_{i}^{a}$ (or $\tilde{t}_{i}^{b}$ ). In the following, the superscripts $a$ or $b$, referring to the grains that form the considered interface, will be discarded for the sake of readability in the expression of the displacement jump and interface tractions, if it does not generate ambiguity. 


\subsection{Interface status}

It is assumed that interface pairs can be in one of the following regions:

- Intact or pristine region $\mathscr{I} \mathscr{R}$ : these pairs are still in the pristine condition. The interface tractions have never exceeded the threshold that defines the onset of damage and no separation is allowed between the grain surfaces. Perfect bonding conditions are directly enforced through displacement compatibility and traction equilibrium equations.

- Damaged or cohesive region $\mathscr{D} \mathscr{R}$ : these pairs have experienced tractions over a certain threshold value, and have started accumulating damage. Tractions equilibrium always holds, but the grain surfaces are now allowed a separation. The interface tractions depend now on the accumulated damage and displacement jump, according to a certain irreversible cohesive law. The pairs entering this zone begin the process that will lead to failure, i.e. cracking, of the interface.

- Failed or cracked region $\mathscr{F} \mathscr{R}$ : these interface pairs have completed the damaging process and have developed a free crack. Their status is now governed by the laws of frictional contact mechanics: they can be either separated or in contact and if they are in contact they will be either in slip or in stick status.

\subsection{Interface equations}

In this section, the equations describing the state of the interface pairs, in any of the aforementioned regions, are introduced in some detail.

Before, however, it is useful to give a general premise about their structure. In the present formulation, each interface pair carries six equations. In the pristine state, there are three continuity equations, expressing identity of displacement components, and three equilibrium equations, expressing identity of traction components. During the interface evolution, the three traction equilibrium equations do not change. On the contrary, the continuity equations are replaced by the cohesive separation-traction laws during the damage onset and subsequently by the frictional contact equations, when failure is reached. In the following, only the three equations that will be changing are discussed, in the different cases, while equilibrium equations must be satisfied throughout the analysis.

Moreover, especially for contact analysis, it is worth making a conceptual distinction between conditions and related equations: some conditions must be satisfied by the interface pair solution displacements and tractions, when the related equations are forced in the system with reference to the considered pair; if the conditions are violated, then another consistent set of equations must be introduced into the system, with reference to the pair, and the related conditions must be satisfied. A consistent solution is reached when conditions and equations are consistent for all the interface pairs. These concepts will be further developed in the following.

\subsubsection{Interface continuity equations}

For an interface pair in the pristine state, $\mathscr{P} \in \mathscr{I} \mathscr{R}$, the following compatibility and equilibrium equations hold

$$
\begin{aligned}
& \tilde{u}_{i}^{a}+\tilde{u}_{i}^{b}=-\delta \tilde{u}_{i}^{a b}=0 \\
& \tilde{t}_{i}^{a}-\tilde{t}_{i}^{b}=0 \\
& i=1,2,3 \quad \text { on } \quad \mathscr{I}_{a b} \equiv \mathscr{G}_{a} \cap \mathscr{G}_{b}
\end{aligned}
$$


The local traction components $\tilde{t}_{i}^{a}=\tilde{t}_{i}^{b}=\tilde{t}_{i}$, at a point $P \in \mathscr{F}_{q}^{a} \subset \mathscr{G}_{a}$, can be naturally used to express the tangential and normal tractions for the pair. The local normal and tangential interface tractions are defined by

$$
\tilde{\mathbf{t}}_{n}=(\tilde{\mathbf{t}} \cdot \tilde{\mathbf{n}}) \tilde{\mathbf{n}}=\left[\begin{array}{c}
0 \\
0 \\
\tilde{t}_{3}
\end{array}\right] \quad \tilde{\mathbf{t}}_{s}=\tilde{\mathbf{t}}-\tilde{\mathbf{t}}_{n}=\left[\begin{array}{c}
\tilde{t}_{1} \\
\tilde{t}_{2} \\
0
\end{array}\right]
$$

and their moduli are given by $t_{n}=\tilde{t}_{3}$ and $t_{s}=+\left(\tilde{t}_{1}^{2}+\tilde{t}_{2}^{2}\right)^{1 / 2}$. It is worth noting that, in the above definition, unlike $t_{s}, t_{n}$ carries a sign. The sign of $t_{n}$ has an important role in the development and evolution of the intergranular damage: when $t_{n}>0$, then the interface experiences tensile tractions, that contribute to the spread of damage; if $t_{n}<0$, then the interface sees a compressive load, that does not contribute to the evolution of damage.

The normal and tangential interface tractions are used to define the effective traction

$$
t_{e}=\left[\left\langle t_{n}\right\rangle^{2}+\left(\frac{\beta}{\alpha} t_{s}\right)^{2}\right]^{\frac{1}{2}}
$$

where $\langle\circ\rangle$ denote the Mc-Cauley brackets, defined by $\langle x\rangle=\max (0, x)$, and $\alpha$ and $\beta$ are suitable coefficients weighing the relative contribution of the opening and sliding modes in the damage process. Again, from the above definition, it can be noticed that only tensile normal tractions contribute to the value of the effective traction. An interface pair $\mathscr{P} \in \mathscr{I} \mathscr{R}$ remains in the pristine status until $t_{e}<T_{\max }$, where $T_{\max }$ defines a threshold value for damage initiation.

\subsubsection{Damaged interface: cohesive traction-separation laws}

When the effective traction exceeds $T_{\max }$, often referred to as cohesive strength, the node pair $\mathscr{P}$ enters the damaged region

$$
t_{e} \geq T_{\max } \quad \Rightarrow \quad \mathscr{I} \mathscr{R} \rightarrow \mathscr{P} \rightarrow \mathscr{D} \mathscr{R}
$$

and an irreversible extrinsic cohesive law, or traction-separation law (TSL), $\tilde{t}_{i}=f_{i}\left(\mathscr{H}_{d}, \delta \tilde{u}_{j}\right)$ is introduced, to link the pair tractions and displacement jumps: $\mathscr{H}_{d}$ symbolizes the dependence on the history of the decohesion process, thus giving the cohesive law the character of irreversibility; the adjective extrinsic, on the other hand, refers to the fact that the traction-separation laws are introduced only after a certain threshold for the effective traction has been overcome.

To follow the evolution of damage, according to Ortiz and Pandolfi [59], a non-dimensional effective opening displacement

$$
d=\left[\left\langle\frac{\delta u_{n}}{\delta u_{n}^{c r}}\right\rangle^{2}+\beta^{2}\left(\frac{\delta u_{s}}{\delta u_{s}^{c r}}\right)^{2}\right]^{\frac{1}{2}}
$$

is introduced, where $\delta u_{n}$ is defined by Eq.(14), $\delta u_{s}=\left(\delta \tilde{u}_{1}^{2}+\delta \tilde{u}_{2}^{2}\right)^{1 / 2}$ and $\delta u_{n}^{c r}$ and $\delta u_{s}^{c r}$ denote the critical values of the normal and sliding displacement jumps at which interface failure occurs, in the case of pure Mode I or Mode II loading respectively. The parameter $\beta$ assigns a different weight to the two modes, in the mixed-mode case. The effective opening displacement can assume values $0 \leq d \leq 1$, where $d=1$ implies the complete failure, or decohesion, of the 
considered pair.

The formulation of the cohesive law can be derived from the introduction of a scalar potential: in this work this derivation is shown; eventually, however, it will be shown how a simple modification of the TSLs removes the hypothesis of existence of the cohesive potential, allowing for the energetic path dependence of the work of decohesion, and then the energetic differentiation between mode I and mode II failures. Following Ortiz and Pandolfi [59], the following potential is introduced

$$
\phi=\phi\left(\delta u_{n}, \delta u_{s}, \mathbf{q}\right)
$$

where $\mathbf{q}$ represents a suitable collection of internal variables describing the irreversible processes attending the decohesion, and whose evolution is governed by some kinetic relationship of the form

$$
\dot{\mathbf{q}}=\psi\left(\delta u_{n}, \delta u_{s}, \mathbf{q}\right) .
$$

The cohesive traction-separation law can be then derived from the potential (20) through the relationships

$$
\tilde{t}_{i}=\frac{\partial \phi}{\partial \delta \tilde{u}_{i}} \quad i=1,2,3 \quad \text { or } \quad t_{n}=\frac{\partial \phi}{\partial \delta u_{n}}, \quad t_{s}=\frac{\partial \phi}{\partial \delta u_{s}} .
$$

Following Espinosa and Zavattieri [62], Sfantos and Aliabadi [69] used a potential of the form

$$
\phi(d)=\delta u_{n}^{c r} \int_{0}^{d} t_{e}(\eta) d \eta
$$

where $d$ is the current effective opening displacement defined by Eq.(19) and

$$
t_{e}(\eta)=T_{\max } \frac{1-d^{*}}{d^{*}} \eta \quad \text { with } \quad d^{*}=\max _{\mathscr{H}_{d}}\{d\} \in[0,1]
$$

where $d^{*}$ is a monotonically increasing parameter, given by the maximum value that the effective opening displacement $d$ reaches during the loading history $\mathscr{H}_{d}$ of the considered pair: it plays the role of a state variable that accounts for the evolution of the degradation process and Eq.(24b) is the kinetic relationship governing its evolution, see Eq.(21). For an undamaged pair $d^{*}=0$, while $d^{*}=1$ for a complete failed pair: in this case the considered pair exits the cohesive zone and a microcrack is introduced. In other words, $d^{*}$ is the variable that accounts for the accumulation of damage for the node pair. It is worth noting that unloading and reloading take place in the range $0 \leq d \leq d^{*}$, while loading implies $d^{*}=d$. More precisely

$$
d=d^{*} \text { and } \dot{d}>0 \text { loading; } \quad 0 \leq d \leq d^{*} \text { and } \begin{cases}\dot{d}<0 & \text { unloading } \\ \dot{d}>0 & \text { reloading }\end{cases}
$$

where the overdot denotes the derivative with respect to a generic load factor $\lambda$, during the loading history.

The cohesive tractions are calculated using Eqs.(22) with the potential defined by Eqs.(23-24) and taking into account the definition of effective opening displacement, Eq.(19). The following expressions are obtained in this case for the normal and tangential traction components

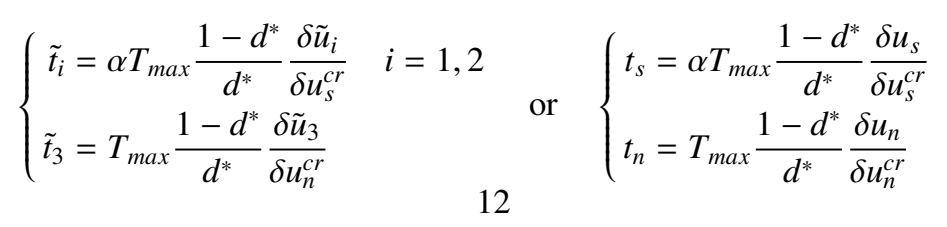


where the relationship

$$
\alpha=\beta^{2} \frac{\delta u_{n}^{c r}}{\delta u_{s}^{c r}}
$$

holds: this relationship is of particular relevance, as its enforcement guarantees the existence of the cohesive potential (23), and then the independence of the cohesive fracture energy from the opening path.

To fully understand this aspect, let us consider the opening of a cohesive surface along a direction that forms a constant angle $\phi$ with the normal to the cohesive surface itself. In this case

$$
\delta u_{n}=u \cos (\phi) \quad \delta u_{s}=u \sin (\phi)
$$

where $u$ is a loading parameter. It is easy to show that the work of separation is given by

$$
G(\phi)=\int_{0}^{u_{c r}} t_{n}(u) d \delta u_{n}+t_{s}(u) d \delta u_{s}=\frac{T_{\max }}{2} \frac{\frac{\cos ^{2}(\phi)}{\delta u_{n}^{c r}}+\alpha \frac{\sin ^{2}(\phi)}{\delta u_{s}^{c r}}}{\left(\frac{\cos (\phi)}{\delta u_{n}^{c r}}\right)^{2}+\beta^{2}\left(\frac{\sin (\phi)}{\delta u_{s}^{c r}}\right)^{2}}
$$

and, in particular, that

$$
G_{I}=G(0)=\frac{1}{2} T_{\max } \delta u_{n}^{c r} \quad G_{I I}=G\left(\frac{\pi}{2}\right)=\frac{\alpha}{2 \beta^{2}} T_{\max } \delta u_{s}^{c r} .
$$

It can be shown that, if the relationship (27) holds, then $G(\phi)=G(0) \forall \phi \in[0, \pi / 2]$ and, in particular, $G_{I}=G_{I I}$. In other words, if a potential traction-separation law is formulated, it is not possible to distinguish energetically between pure mode I and pure mode II, as the fracture energy is required to be independent from the fracture path. However, as pointed out by some authors $[92,93]$, it is reasonable to assume that the work of decohesion should be path dependent, as the energy dissipated in a fracture process depends on some microstructural details that inherently make mode II different from mode I, at least at a macroscopic level. In the framework introduced above, the fracture energy path-dependency can be achieved by simply discarding Eq.(27) and assigning suitable independent values to the constants $\alpha$ and $\beta$ : this corresponds to relaxing the requirement of existence of a well defined cohesive potential; the traction-separation laws are then directly given by Eqs.(26) and not derived from a given potential. Therefore, in this work, the cohesive traction-separation laws are specified by Eqs.(26), where the constants $\alpha$ and $\beta$ are given independently from each other, but in such a way to ensure the desired $G_{I I} / G_{I}$ ratio between mode II and mode I fracture energies: they are not required to fulfill Eq.(27). The laws obtained in this way are similar to those presented by Snozzi and Molinari [93], who used the constants in a slightly different way. For the case $G_{I I} / G_{I}=2$, Eq.(29) is plotted in Fig.(4), where the energetic contribution from the opening term $\delta u_{n}$ is distinguished from that coming from the sliding term $\delta u_{s}$, for $\phi \in[0, \pi / 2]$.

The traction-separation laws (26) are valid in all the loading cases, i.e. whether the considered pair is in loading, unloading or reloading. During unloading and reloading $d^{*}$ remains constant, its value being given by the higher effective opening displacement reached during the loading history, before unloading took place; during unloading and reloading, the traction components (26) become then linear with respect to the current value of the displacement jumps. Moreover, Eqs.(26) express an isotropic cohesive law, as the cohesive tangential tractions depend on the tangential displacement jumps according to the same law in any direction on the contact plane. It is worth noting that the normal cohesive traction component $t_{n}$ is given by Eqs.(26) only when 
a normal opening displacement $\delta u_{n}$ is present. The condition $\delta u_{n} \geq 0$, that expresses the physical impossibility of grain interpenetration, must always be verified. In the case of compressive loading, the equation $\delta u_{n}=0$ must be enforced, instead of Eq.(26); the pair moves into the pure sliding loading mode and the normal traction component $t_{n}$ is provided by the solution of the microstructural problem.

The normal and tangential components of the tractions-separation law are plotted in Fig.(5).

\subsubsection{Interface failure: frictional contact analysis}

Once $d^{*}=1$, the considered pair $\mathscr{P}$ leaves the damaged region and fails, forming a microcrack and entering the cracked zone:

$$
d^{*}=1 \quad \Rightarrow \quad \mathscr{D} \mathscr{R} \rightarrow \mathscr{P} \rightarrow \mathscr{F} \mathscr{R}
$$

Upon interface failure, the laws of frictional contact mechanics enter the formulation and govern the evolution of the interface pair $[94,95,96]$. The Coulomb frictional law is implemented in this work. Once a micro-crack has started, the two crack surfaces can be separated or in contact. In the case of separation, the condition $\delta u_{n}>0$ must always be satisfied, and the following equations hold for the traction components

$$
\tilde{t}_{i}=0 \quad i=1,2,3 \quad \text { or } \quad t_{n}=0, \quad t_{s}=0 .
$$

If the crack surfaces are in contact, then they can stick or slide. In the case of contact, the condition $t_{n} \leq 0$ must always be satisfied, while the equation $\delta u_{n}=0$ is directly enforced in the system of equations, either in case of stick or slip.

If the surfaces are in contact and the condition $\left|t_{s}\right|<\mu\left|t_{n}\right|$ holds, then the pair is said to be in stick status and the following equations hold:

$$
\left\{\begin{array} { r l } 
{ \Delta \delta \tilde { u } _ { i } } & { = 0 \quad i = 1 , 2 } \\
{ \delta \tilde { u } _ { 3 } } & { = 0 }
\end{array} \quad \text { or equivalently } \left\{\begin{array}{rl}
\Delta \delta u_{s} & =0 \\
\delta u_{n} & =0
\end{array}\right.\right.
$$

where the symbol $\Delta$ denotes the increment of a quantity between load steps: no variation of the displacement jump is allowed if the pair is in stick condition.

If the stick condition $\left|t_{s}\right|<\mu\left|t_{n}\right|$ cannot be satisfied, then the relationship $\left|t_{s}\right|=\mu\left|t_{n}\right|$ must be enforced and the pair is said to be in slip status. In this case, the relative displacements $\delta \tilde{u}_{1}$ and $\delta \tilde{u}_{2}$ can evolve and the following equations hold:

$$
\left\{\begin{aligned}
\tilde{t}_{1}+\mu \tilde{t}_{3} \cos (\theta) & =0 \\
\tilde{t}_{2}+\mu \tilde{t}_{3} \sin (\theta) & =0 \\
\delta \tilde{u}_{3} & =0
\end{aligned}\right.
$$

where $\theta$ denotes the slip angle, defined in the plane $\left\{\tilde{x}_{1} \tilde{x}_{2}\right\}$ by $\tan (\theta)=\delta \tilde{u}_{2} / \delta \tilde{u}_{1}$ : this angle is not a priori known and its value must be computed iteratively in the solution process, as it will be explained in the next section. From the above definition of slip angle and from Eqs.(34), it is apparent that the two vectors $\delta \tilde{\mathbf{u}}_{s}$ and $\tilde{\mathbf{t}}_{s}$ should have the same direction in the contact plane. However, since the equation $\tan (\theta)=\delta \tilde{u}_{2} / \delta \tilde{u}_{1}$ is not directly implemented into the system, this requirement must be assessed once the numerical solution of the problem is available. Let us define $\beta_{s}$ as the angle between $\delta \tilde{\mathbf{u}}_{s}$ and $\tilde{\mathbf{t}}_{s}$ on the contact plane. The slip condition is violated if $\left|\beta_{s}\right| \geq \pi / 2$, i.e. $\tilde{\mathbf{t}}_{s} \cdot \delta \tilde{\mathbf{u}}_{s} \leq 0$ : if this happens, then the pair cannot be in slip and it is put in stick status. On the other hand, if $\left|\beta_{s}\right|<\pi / 2$ but $\left|\beta_{s}\right|>0$, then the slip angle must be suitably adjusted. 
Figure 1: Voronoi tessellations generated with Voro++ [87]: a) 1000-grain 3D tessellation; b) 200-grain pseudo-3D tessellation. Some transparency is given to the surface of the grains to give an insight into the complexity of the internal structure.

Figure 2: Local reference system on a grain face: displacements and tractions of points belonging to the face $\mathscr{F}_{j}^{k}$ are expressed in the reference system $\left\{\tilde{x}_{1} \tilde{x}_{2} \tilde{x}_{3}\right\}_{j}^{k}$.

Figure 3: Interface between the two contiguous grains $\mathscr{G}_{a}$ and $\mathscr{G}_{b}$ and opposite local reference systems attached to the faces in contact.

Figure 4: Fracture energy for a decohesion process along a direction forming a constant angle $\phi$ with the direction normal to the cohesive surface, in the case $G_{I I} / G_{I}=2$.

Figure 5: $(a)$ : Graphical representation of the normal and tangential cohesive traction-separation laws, $t_{n}\left(\delta u_{n}, \delta u_{s}\right)$ and $t_{s}\left(\delta u_{n}, \delta u_{s}\right) ;(b)$ : Intersections of the cohesive surfaces with the planes $\delta u_{s}=$ const and $\delta u_{n}=$ const are visualized. 


\subsubsection{Grains interface under compressive-sliding load: cohesive-frictional laws}

In the depicted framework, a particular aspect deserves some attention. As explained above, when an interface between two grains fails, the cohesive laws no longer apply and the frictional contact analysis is used instead. This on-off switch between cohesive and frictional contact laws is acceptable in the case of truly mixed-mode loading, i.e. when a normal traction component $t_{n}>0$ exists. However, when the interface is subjected to a compressive-sliding load, see Fig.(6), the sudden transition between the two regimes can give raise to some physically inconsistent behavior as well as to some numerical problems. As pointed out by Snozzi and Molinari [93], this aspect has often been overlooked.

To understand the issue, let us consider the interface between the two grains shown in Fig.(6a): the two grains are subjected, in displacement control, to a compressive load $\lambda \Delta v$ and to a sliding load $\lambda \Delta u$, where $\lambda$ denotes the load factor. The interface is initially undamaged and, as shown in Fig.(6b), the aggregate is able to carry elastically a load until a cohesive process is started at the interface; at this point, since a compressive load is present, the impenetrability is directly enforced in the system, while the tangential components of tractions are given by the corresponding term in Eqs.(26). As the interface damage grows, the load-carrying capability of the system is progressively reduced: at this point, if the model implements a switch between cohesive and friction laws, then the tangential cohesive tractions must go to zero at $d^{*}=1$, i.e. upon interface failure, before the laws of contact mechanics can be applied. The trend of the tangential traction, in this case, is given by the lower (blue) curve of Fig.(6b). After interface failure, contact mechanics apply and the contact algorithm correctly predicts a stick condition immediately after failure, to avoid a sudden discontinuity of the tangential traction during the loading process, followed, eventually, by the slip of the interface, for which $t_{t}=\mu\left|t_{n}\right|$.

However, the prediction that, under this loading condition, the interface loses completely its load-carrying capability, before retrieving some of it through the friction $\mu$, appears slightly inconsistent. Moreover, the presence of the cusp at the transition between cohesive and contact state, and the possibility of a traction jump if the load factor is not correctly updated, is likely to spark numerical instabilities, especially when multi-body frictional contact analysis is considered. To avoid such behavior, in this work, the presence of some friction is assumed during the decohesion process, if it happens in compression: this corresponds to assuming a continuous transition between cohesive and contact state.

The gradual transition to the contact state is achieved by introducing some frictional terms depending on the damage level in the traction-separation laws. In this case, the tangential components of traction are expressed by means of the following cohesive-frictional laws

$$
\left\{\begin{array}{l}
\tilde{t}_{1}=\alpha T_{\max } \frac{1-d^{*}}{d^{*}} \frac{\delta \tilde{u}_{1}}{\delta u_{s}^{c r}}-\chi\left(d^{*}\right) \mu \tilde{t}_{3} \cos (\theta) \\
\tilde{t}_{2}=\alpha T_{\max } \frac{1-d^{*}}{d^{*}} \frac{\delta \tilde{u}_{2}}{\delta u_{s}^{c r}}-\chi\left(d^{*}\right) \mu \tilde{t}_{3} \sin (\theta)
\end{array}\right.
$$

where $\theta$ is the slip angle and $\chi\left(d^{*}\right)$ is a suitable function modelling the soft transition from zero to full friction; in this work $\chi(d)$ is phenomenologically assumed to be

$$
\chi(d)=\frac{1}{2}\left\{1+\tanh \left[\frac{d-\bar{d}}{d(1+\xi-d)}\right]\right\}
$$

where $0<\bar{d}<1$ and $0<\xi \ll 1$ are parameters that enable one to modify, to some extent, the shape of the curve $\chi(d)$, see Fig.(7). 
If Eqs.(35)and Eq.(36) are implemented, the trend of the tangential tractions at the interface between the two grains shown in Fig.(6a) is given by the upper (red) curve in Fig.(6b). In the figure, the value of the intergranular tangential traction is given as a function of the multiplicative load factor $\lambda$ and it is apparent how the introduction of the frictional terms smooths the transition to the frictional contact, favoring a gradual evolution from cohesive debonding to frictional slip. The grey area between the two curves in Fig.(6) represents the energy dissipated by the friction during the decohesion process.

\subsection{Numerical aspects: mesh requirements}

The mesh of the polycrystalline microstructure is generated as explained in Appendix B. However, some additional considerations are required and care must be taken in the mesh preparation to fulfill the conditions for element-size independency and reproducibility of the solution. As investigated by Tomar et al. [97] and Espinosa and Zavattieri [63], and as reported by Sfantos and Aliabadi [69], factors influencing the solution mesh independency and reproducibility are: $a$ ) the initial macroscopic stiffness reduction resulting from the cohesive separation along the grain boundary, when the initial slope of the cohesive law is finite; $b$ ) the characteristic element length $\xi_{e}$, that must be fine enough to resolve the strain and stress distributions inside the cohesive zone. In the proposed formulation, the initial stiffness of the cohesive law is infinite, as perfect bonding between pair nodes is enforced directly in the system, Eq.(15), until the fulfillment of condition (18) triggers the cohesive separation process: then the first factor does not affect the solution process in the present case. The second factor is however very relevant and the condition

$$
\xi_{e} \ll L_{\mathscr{C} \mathscr{Z}}
$$

must be fulfilled, where $L_{\mathscr{C} \mathscr{Z}}$ denotes the length of the cohesive zone, that must be estimated in order to make Eq.(37) effective. An estimate of the cohesive zone size, for a linearly softening cohesive law, as the one adopted in the present study, was provided by Rice [98] as

$$
L_{\mathscr{C} \mathscr{Z}} \approx \frac{\pi}{2}\left(\frac{K_{I C}}{T_{\max }}\right)^{2}
$$

and it was used by Espinosa and Zavattieri in [63]; in the previous equation $K_{I C}$ is the material fracture toughness in mode I, and $T_{\max }$ is the threshold value entering condition (18), i.e. the strength of the cohesive grain boundary under pure normal separation. Another estimate for cohesive laws derived from a potential is given in [97] as

$$
L_{\mathscr{C} \mathscr{Z}} \approx \frac{9 \pi}{32} \frac{E}{\left(1-v^{2}\right)} \frac{\phi_{0}}{T_{\max }^{2}}
$$

where $E$ is the material Young modulus, $v$ is the Poisson ratio and $\phi_{0}$ is the amount of work required to separate completely a unit surface starting from undamaged state.

\section{Micro-damage and micro-cracking tracking}

The algorithm employed to track the evolution of micro-damage and micro-cracks along the intergranular boundaries is described. Some details about the choice of the algorithm parameters are given. The practical numerical implementation adopted in this work is given in Appendix C. 


\subsection{Microstructural evolution algorithm}

Given a polycrystalline aggregate and a consistent set of boundary conditions, the internal evolution of the microstructure is determined by the increment of the external loads, whose intensity can be generally expressed in terms of a load factor $\lambda$. The microstructural evolution is tracked by solving the boundary value problem for a discrete set of values $\lambda_{k}\left(k=0, \ldots, N_{\lambda}\right)$

$$
\mathbf{A}\left(\mathscr{H}_{k}\right) \mathbf{X}_{k}=\mathbf{Y}\left(\lambda_{k}\right)
$$

where $\mathscr{H}_{k}$ symbolizes the internal state of the microstructure. Once the solution of the system is obtained for $\lambda_{k}$, the load factor is incremented by a suitably chosen amount $\Delta \lambda_{k}$ and a new solution of system (40) is sought for $\lambda_{k+1}=\lambda_{k}+\Delta \lambda_{k}$. It is worth emphasizing that, in Eq.(40), solution $\mathbf{X}_{k}$ and the microstructural state $\mathscr{H}_{k}$ must be mutually consistent: this requires the numerical solution to fulfill the interface conditions holding: pristine, cohesive or contact state. This consideration is the basis of the solution strategy proposed next.

\subsubsection{Load increment}

Let us assume that a solution of the system (40) has been obtained for $\lambda_{k}$ and that a solution corresponding to $\lambda_{k+1}$ is sought. Once the increment $\Delta \lambda_{k}$ has been determined, the right-hand side of the system is updated by determining $\mathbf{Y}\left(\lambda_{k+1}\right)$ : if no interface pair has failed yet, this step does not require any special consideration and $\mathbf{Y}\left(\lambda_{k+1}\right)$ is simply obtained by scaling $\mathbf{Y}\left(\lambda_{k}\right)$; when micro-cracks are formed and the frictional contact analysis has started, if interface pairs in contact-stick state exist, then the part of $\mathbf{Y}\left(\lambda_{k}\right)$ corresponding to the boundary integral equations will be updated by simple scaling, while the part corresponding to the interface equations will be kept constant. Upon update of the right-hand side, the new system is given by

$$
\mathbf{A}\left(\mathscr{H}_{k+1}^{o}\right) \mathbf{X}_{k+1}^{o}=\mathbf{Y}\left(\lambda_{k+1}\right),
$$

where the matrix $\mathbf{A}\left(\mathscr{H}_{k+1}^{o}\right)=\mathbf{A}\left(\mathscr{H}_{k}\right)$ has not been updated yet, will be solved: this simply involves the solution of a linear system and, if no failed pairs in contact-stick state exist, the solution $\mathbf{X}_{k}^{o}$ can be found by simply scaling $\mathbf{X}_{k}$. At this point an iteration is started with the aim of finding the new system solution $\mathbf{X}_{k+1}$ satisfying Eq.(40) for $k+1$ : in Eq.(41), $\mathscr{H}_{k+1}^{i}$ and $\mathbf{X}_{k+1}^{i}$ denote respectively the internal state and the solution at the $i$-th iteration of the $(k+1)-t h$ increment.

\subsubsection{Iterative search}

The iterative search of the solution for the current load increment is carried forward until convergence is reached. In general, at each iteration, some interface pairs are in the pristine state $\mathscr{I} \mathscr{R}$, some are in the cohesive zone $\mathscr{D} \mathscr{R}$ and others belong to the cracked interface $\mathscr{F} \mathscr{R}$. The generic solution $\mathbf{X}_{k+1}^{i}$ at the $i-t h$ iteration provides the complete set of interface displacement jumps and tractions: the convergence of the iterative procedure is checked by assessing whether any state violation exists for any interface pair, be it in the pristine state, in the cohesive zone or in the failed region.

- The intact pairs are checked to assess whether the effective traction defined by Eq.(17) is below the threshold value $T_{\max }$. A state violation occurs if $T_{\max }$ is overcome: in this case, 
the displacement continuity equations do not hold anymore and the interface equations corresponding to the considered pair in the coefficient matrix $\mathbf{A}\left(\mathscr{H}_{k+1}^{i}\right)$ must be updated by implementing the cohesive laws (26) with an initial guess value $d_{\text {start }}^{*}$ of the damage.

- The damaged pairs are assessed by comparing the current value of the effective displacement $d_{k+1}^{i}$, Eq.(19), with both the value of the effective displacement computed at the previous iteration $d_{k+1}^{i-i}$ and the damage $d_{k}^{*}$ accumulated by the pair (here subscripts and superscripts refer to the increment and iteration respectively). The assessment of violations for pairs in the damaged state is quite delicate, because different potential situations might arise and must be appropriately addressed. A clear distinction must be initially stated between the damage $d_{k}^{*}$ and the values $d_{k+1}^{i}$ that are found during the iterations: the damage expresses the state in which the considered pair is and can be numerically seen as the convergence value of the series of values $\left\{d_{k}^{1}, \ldots, d_{k}^{i} \ldots, d_{k}^{n}\right\}$ at the previous increment; the values $d_{k+1}^{i}$ are some attempt values that must be assessed until convergence is reached and the state of the pair can be eventually updated with the new value $d_{k+1}^{*}$.

Now, at the first iteration of the increment $\lambda_{k+1}$, the cohesive law of the considered pair is implemented with $d^{*}=d_{k}^{*}$. When the iteration is performed, a new value of the effective displacement $d_{k+1}^{o}$ is obtained: if $d_{k+1}^{o}>d_{k}^{*}$, then the pair is in loading state and its cohesive law has to be updated assuming $d_{k+1}^{o}$ as an attempt value for the damage in Eqs.(26); if $d_{k+1}^{o} \leq d_{k}^{*}$, then the pair is either in unloading or reloading state and its cohesive law does not need to be updated. Upon update of the matrix $\mathbf{A}$, a new solution is computed and a new value $d_{k+1}^{1}$ is provided for the considered pair: if it was in loading state, this value is compared with $d_{k+1}^{0}$ and if $\left|d_{k+1}^{1}-d_{k+1}^{0}\right|>\varepsilon_{d}\left|d_{k+1}^{0}\right|$, where $\varepsilon_{d}$ is a previously set tolerance, then the cohesive law is updated with the new value $\max \left(d_{k+1}^{1}, d_{k}^{*}\right)$. The convergence for a pair in loading state is reached when

$$
\left|d_{k+1}^{i+1}-d_{k+1}^{i}\right| \leq \varepsilon_{d}\left|d_{k+1}^{i}\right|
$$

on the other hand, since unloading or reloading do not require the update of the cohesive law, they do not affect the convergence.

It is worth noting that, if the considered damaged pair is subjected to a compressive load, then the impenetrability condition $\delta u_{n}=0$ is directly enforced in the system, together with the Eqs.(35) expressing the cohesive-frictional law introduced in Section 3.3.4.

- Finally, the state of the pairs in the failed region is assessed. For a failed pair $d^{*}=1$ and the frictional contact analysis started. The assessment is carried out by checking whether any violation of the conditions holding for the assumed contact status exists. Different cases can be met.

- If the pair is assumed to be in separation at the current iteration, which means that Eqs.(32) are currently implemented in the matrix $\mathbf{A}\left(\mathscr{H}_{k+1}^{i}\right)$, then the value of $\delta u_{n}$ is checked, to detect any interpenetration: if $\delta u_{n}<0$, then the separation condition is violated and the contact state of the pair must be updated; in this specific case, contact-stick state is assumed and the corresponding Eqs.(33) are implemented into the system matrix. 
- If the pair is assumed to be in contact, either stick or slip, then the value of the normal traction $t_{n}$ is checked first: if $t_{n}>0$, then the contact assumption is violated and separation must be enforced. If no contact mode violation is detected, then the assessment is carried on, to check whether any stick or slip status violation exists. If the pair is in stick status, then the condition $\left|t_{s}\right|<\mu\left|t_{n}\right|$ is checked and, if violated, the pair is put in slip and Eqs.(34) are implemented with an initial guess value of the slip angle $\theta=\arctan \left(\tilde{t}_{2} / \tilde{t}_{1}\right)_{k+1}^{i}$. If the pair is assumed to be in slip status, then the condition $|\beta|<\pi / 2$ is checked, where $\beta$ is the angle between $\delta \tilde{\mathbf{u}}_{s}$ and $\tilde{\mathbf{t}}_{s}$ in the contact plane, as seen in Section (3.3.3). If a violation is detected, then the pair is put in stick status; on the other hand, if $|\beta|<\pi / 2$ but $|\beta|>\varepsilon_{\beta}$, where $\varepsilon_{\beta}$ is a previously set numerical tolerance, then the slip angle in Eqs.(34) must be adjusted. In this work, the new slip angle is chosen as $\theta=\left(\beta_{t}+\beta_{\delta u}\right) / 2$, where $\beta_{t}=\arctan \left(\tilde{t}_{2} / \tilde{t}_{1}\right)_{k+1}^{i}$ and $\beta_{\delta u}=\arctan \left(\delta \tilde{u}_{2} / \delta \tilde{u}_{1}\right)_{k+1}^{i}$ are the angles formed by the tangential traction and displacement jump respectively with the axis $\tilde{x}_{1}$.

The convergence is reached when no violations are detected for any interface pair and no equation updates are then brought into the system matrix, so that $\mathbf{A}\left(\mathscr{H}_{k+1}^{i}\right)=\mathbf{A}\left(\mathscr{H}_{k+1}^{i+1}\right)$. The system solution $\mathbf{X}_{k+1}$ is then determined and, if needed, another load increment can be applied to the system, starting a new iterative search. The described solution procedure is illustrated in Fig.(8).

\subsection{Parameters set up}

As seen above, several different parameters enter the formulation: the load increment, expressed in terms of $\Delta \lambda_{k}$; the initial guess value $d_{s t a r t}^{*}$ for the damage of an interface pair that has just entered the cohesive zone; the tolerance $\varepsilon_{d}$ used in the assessment of the iteration convergence for the damaged pairs; the angle $\varepsilon_{\beta}$, used to assess the fulfillment of the slip condition. To make the analysis effective, both in terms of accuracy and performance, these parameters must be carefully tuned.

The load increment $\Delta \lambda_{k}$ plays a crucial role in the algorithm for the analysis of the microstructural damage evolution. In principle, it should be chosen small enough to induce a state change in a small number of interface pairs, so that few equations would need to be changed in the system and few iterations would be needed to converge to a consistent solution. The choice of an inconsistently large load increment leads generally to an inconsistent solution, as the microstructural evolution leading from the state $\mathscr{H}_{k}$ to the state $\mathscr{H}_{k+1}$ is missed. However, an excessively small load increment could make the analysis excessively time consuming and might produce a large amount of not always useful information. Ideally, $\Delta \lambda_{k}$ should be selected considering the change induced in a certain number of interface elements at a time, rather than nodes.

In this work $\Delta \lambda_{k}$ is chosen adaptively on the basis of the number of iterations $N_{i t e r}^{k-1}$ needed to reach convergence at the previous load increment. A relationship of the following form is implemented

$$
\Delta \lambda_{k}=f\left(\frac{N_{\text {iter }}^{k-1}}{N_{\max }}\right) \Delta \lambda_{k-1}
$$

where $f\left(N_{i t e r}^{k-1} / N_{\max }\right)<1$ if $N_{i t e r}^{k-1} / N_{\max }$ is closer to 1 and $f\left(N_{i t e r}^{k-1} / N_{\max }\right)>1$ if $N_{i t e r}^{k-1} / N_{\max }$ is closer to 0 . Moreover, to avoid pathological situations, the following constraint is always enforced

$$
\Delta \lambda_{\min } \leq \Delta \lambda_{k} \leq \Delta \lambda_{\max }
$$


where $\Delta \lambda_{\min }$ is chosen large enough to avoid the stagnation of the solution and $\Delta \lambda_{\max }$ is chosen small enough to avoid missing important features of the solution.

The initial guess value $d_{\text {start }}^{*}$ for the damage of a pair just entered in the cohesive state must be chosen small enough to avoid fictitious damage localization at the first damaged interfaces.

Also $\varepsilon_{d}$ must be carefully chosen: on one hand, indeed, $\varepsilon_{d}$ affects the accuracy of the intergranular damage level estimation associated to a certain load factor; on the other hand, it has a direct influence on the number of iterations required for the damage convergence of the cohesive pairs (the lower the value of $\varepsilon$, the higher the number of iterations). The same considerations apply to $\varepsilon_{\beta}$.

In other words, in the set-up of the analysis, there is always a trade off between accuracy and convergence rate, that must be taken into account. In the present study, after careful testing, the following values have been selected for the mentioned parameters: $d_{\text {start }}^{*} \approx 10^{-5}-10^{-4}$, $\varepsilon_{d}=10^{-4}-10^{-3}, \varepsilon_{\beta}=5^{\circ}$.

\section{Micro-damage and micro-cracking simulations}

In this section the results of some numerical simulations are reported and discussed. All the reported tests have been performed on the cxl system of the High Performance Computing facilities at Imperial College London.

\subsection{Numerical estimation of the effective properties of $\mathrm{SiC}$}

Before simulating the micro-cracking initiation and evolution in polycrystalline aggregates, the macroscopic effective properties of silicon carbide ( $\mathrm{SiC})$ are estimated.

The material properties' estimation is based on the following steps: $a$ ) a certain number of different RVE realizations, each subjected to a suitable set of linearly independent boundary conditions, is considered; $b$ ) the boundary value problem for each realization and each set of $\mathrm{BCs}$ is solved with the proposed formulation, in the case of pristine interface (no intergranular damage); c) the stress and strain volume averages for each realization and for each set of BCs are computed through integrals extended to the RVE's external surface only, then taking advantage of the boundary element nature of the formulation itself; $d$ ) the apparent material constants for each realization are estimated from the corresponding sets of volume averaged stresses and strains; $e$ ) the effective properties are obtained as ensemble averages of the previously determined apparent properties, taken over the considered number of different realizations with the same number of grains. For further details the interested reader is referred to the works by Benedetti and Aliabadi $[70,99]$.

The performed analysis takes into account the stochastic nature of the microstructure, in terms of grain size, morphology and orientation. $N_{r}=100$ realizations of aggregates with 150 grains have been generated and analyzed. Each realization differs from the others in terms of both geometry and crystallographic orientation. Given a polycrystalline realization, consisting of $N_{g}$ grains and subjected to a given set of consistent boundary conditions, since the material is supposed to not develop microcracks, stress and strain volume averages can be used to extract the apparent elastic modula, see for example $[3,76,100]$. Kinematic uniform boundary conditions, i.e. linear displacement boundary conditions corresponding to prescribed macro-strains, have been enforced on each simulated realization. Table 5.1 reports the elastic constants for hexagonal 
single crystal $\mathrm{SiC}$, as measured by Arlt and Schodder [101]: these constants define the material of the single grains, which are then given a random orientation in the $3 \mathrm{D}$ space. The values of the effective elastic modula $E$ and $G$ for polycrystalline $\mathrm{SiC}$ have been reported by various authors, see Lambrecht et al. [102] and references therein. In this study, the average values, calculated over $N_{r}=100$ realizations of aggregates with $N_{g}=150$ grains, are $E=456 \mathrm{GPa}$ and $G=193$ $\mathrm{GPa}$, which are in very good agreement with the values $E=448 \mathrm{GPa}$ and $G=192 \mathrm{GPa}$, reported by Carnahan [103], who used low porosity samples and extrapolated the values to zero porosity. The average computed value of the Poisson ratio was $v=0.181$, close to the value $v=0.168$ yielded by Carnahan estimations.

\begin{tabular}{cccccc}
\hline$C_{11}$ & $C_{12}$ & $C_{13}$ & $C_{33}$ & $C_{44}$ & $C_{66}$ \\
\hline 502 & 95 & 96 & 565 & 169 & 203.5 \\
\hline
\end{tabular}

Table 1: Elastic constants for hexagonal single-crystal SiC [GPa], from [101]. The Voigt notation is used.

\subsection{Micro-cracking of pseudo-3D specimens under tensile load}

Next, the intergranular micro-cracking of pseudo-3D (2D columnar) polycrystalline $\mathrm{SiC}$ specimens subjected to tensile load is simulated. These tests have been performed to assess the performance of the developed numerical scheme and to qualitatively compare the obtained results with those reported by Sfantos and Aliabadi [69].

First, the 100-grain configuration shown in Fig.(9a) is simulated: it is worth noting that we are dealing with a three-dimensional geometry obtained by extruding a two-dimensional tessellation; the crystallographic orientation of the grains is however not restrained and remains fully three-dimensional. The average grain size in the $\{x-y\}$ plane is ASTM $G=10$, so that the grain average area is $\bar{A}_{g r}=126 \mu \mathrm{m}^{2}$ [104]. The overall specimen size is $2 \mathrm{~W} \times 2 H \times 2 T$, with $H=2 W, 4 W H=N_{g} \bar{A}_{g r}$ and $2 T=\sqrt{\bar{A}_{g r}}$. The elastic constants of the grains are given in Table 5.1; the macroscopic elastic modulus and Poisson ratio have been selected as $E=448 \mathrm{GPa}$ and $v=0.168$. The fracture toughness of $\mathrm{SiC}$ was assumed $K_{I C}=3 \mathrm{MPa} \mathrm{m}^{1 / 2}$ throughout this study. The interface cohesive-frictional properties for the pseudo-3D specimens under tensile load are summarized in Table 2. It is worth noting that no parameter such as the displacement at the damage initiation point is required in the formulation, as the damage is started only when the value of the effective traction reaches the cohesive strength $T_{\text {max }}$; in other words, the traction-separation laws have infinite initial stiffness and the separation at damage initiation is zero. On the other hand, given the fracture toughness $K_{I C}$, the value of the critical displacement jump $\delta u_{n}^{c r}$ can be inferred considering the equations

$$
G_{I}=\frac{\left(1-v^{2}\right) K_{I C}^{2}}{E}=\frac{1}{2} T_{\max } \delta u_{n}^{c r}
$$

\begin{tabular}{ccccccc}
$T_{\max }[\mathrm{MPa}]$ & $\alpha$ & $\beta$ & $G_{I I} / G_{I}$ & $\mu$ & $\bar{d}_{E q .(36)}$ & $\xi$ \\
\hline 500 & 1 & $\sqrt{2}$ & 1 & 0.05 & 0.8 & 0 \\
\hline
\end{tabular}

Table 2: Sets of cohesive-frictional parameters for the pseudo-3D micro-specimens subjected to tensile load. 
expressing the relationship between the work of separation $G_{I}$ and the fracture toughness $K_{I C}$ in mode I, in the case of plain strain, and that between work of separation $G_{I}$ and the cohesive parameters $T_{\max }$ and $\delta u_{n}^{c r}$. It should be noted that the $G_{I}$ is given by the area under the tractionseparation curve $t_{n}\left(\delta u_{n}\right)$. Moreover, given the parameters $\alpha$ and $\beta$ and the ratio $G_{I I} / G_{I}, \delta u_{s}^{c r}$ can be inferred from Eqs.(30), that hold general validity.

Assuming a coordinate reference system centered at the center of symmetry of the specimen, and with the axes aligned with the specimen edges, as shown in Fig.(9a), the applied boundary conditions are

$$
\begin{array}{clrl}
t_{x}=t_{y}=t_{z}=0(x=\mp W), & t_{x}=t_{z}=0, u_{y}=0 \quad(y=-H), \\
t_{x}=t_{y}=u_{z}=0(z=\mp T), & t_{x}=t_{z}=0, u_{y}=\Delta \mathrm{U} \quad(y=+H) .
\end{array}
$$

The specimen is subjected to a uniform displacement $\Delta \mathrm{U}$ applied over the surface $y=H: \Delta \mathrm{U}$ increases from zero up to the value that causes the complete failure of the specimen.

First, a set of tests has been carried out to assess the mesh independency of the proposed formulation. The microstructure shown in Fig.(9a) has been discretized setting different values for the mesh density parameter $d_{m}$ (see Appendix B): four different meshes, corresponding to the values $d_{m}=0.5,1,1.5,2$, have been prepared. The meshes are shown in Fig.(10) and their main features are reported in Table 3. In the table, $\zeta$ represents a characteristic linear size for the triangular boundary elements: from the reported values it is apparent that both $\zeta_{\text {avg }} \ll L_{\mathscr{C} \mathscr{Z}}$ and $\zeta_{\max } \ll L_{\mathscr{C} \mathscr{Z}}$ are satisfied, so that, according to what has been discussed in Section 3.4, mesh independency is expected. This result is interesting and it strictly holds in the sense shown in the numerical test. However, mesh independency should always be tested and not taken for granted, should the model be extended to more complex constitutive behaviors.

The simulated stress-strain curves for the four different meshes are reported in Fig.(11). As a close examination of the zoomed region highlights, the maximum difference in the predicted stress between the simulated curves, at a given level of strain, is less than 1\%; on the other hand, the maximum difference in the predicted failure strain, between the different meshes, is around $0.6 \%$; moreover all the meshes predict the same failure conditions in terms of micro-cracking patterns. It is worth noting that, for all the reported curves, the linear part corresponds to a material with Young's modulus $E=450, \mathrm{GPa}$, which is consistent with the value known for $\mathrm{SiC}$ and with the value previously computed through numerical homogenization.

Fig.(9b) shows the micro-crack path for the analyzed microstructure: this has been plotted processing the output from the numerical analysis of the finer mesh $\left(d_{m}=2\right)$, but, as already

\begin{tabular}{rcccc} 
Mesh density $-d_{m}$ & 0.5 & 1.0 & 1.5 & 2.0 \\
\hline Number of elements & 2,672 & 3,730 & 7,369 & 12,428 \\
Interface elements & 632 & 826 & 1,638 & 2,752 \\
\hline$\zeta_{\max }[\mu \mathrm{m}]$ & 9.79 & 7.60 & 5.09 & 3.81 \\
$\zeta_{\text {avg }}[\mu \mathrm{m}]$ & 5.68 & 5.18 & 3.83 & 2.99 \\
\hline DoFs & 35,496 & 48,438 & 95,805 & 161,388 \\
Average time per $\Delta \lambda$ & $20 \mathrm{~s}$ & $44 \mathrm{~s}$ & $197 \mathrm{~s}$ & $1,199 \mathrm{~s}$ \\
\hline
\end{tabular}

Table 3: Some statistics about the analyzed meshes for the considered tessellation (number of grains $N_{g}=100$; number of grain interfaces $N_{\text {int } f}=262$ ). $\zeta$ represents a characteristic linear size for the triangular boundary elements; $\Delta \lambda$ denotes the load increment. Each test was performed on a single 12-core node of the cxl HPC system at Imperial College London, with 12-24 GBs of required virtual memory, depending on the system size. 
Figure 6: $(a)$ : Intergranular interface subjected to compressive-sliding load; $(b)$ : Tangential component of traction at the interface, in the cases of cohesive-to-frictional and cohesive-frictional behavior; the grey area accounts for the amount of energy dissipated by the friction during the decohesion process.

Figure 7: Trend of the function $\chi(d)$ used to model the soft transition between cohesive debonding and frictional contact, for different values of the tuning parameters $\bar{d}$ and $\xi$.

Figure 8: Flow chart of the proposed solution strategy.

Figure 9: (a): Schematic of the 100-grain pseudo-3D polycrystalline SiC specimen subjected to uniaxial tensile load; $(b)$ : Micro-cracking pattern for the analyzed microstructure, obtained from the finer mesh $\left(d_{m}=2\right)$. The two frames show the interface damage level one step before and one step after the complete failure. The colour scale has been suitably tailored so as to highlight the presence of little damage just before the complete cracking (for colour interpretation the reader is referred to the electronic version of the paper). The strain levels $\varepsilon$ corresponding to the frames are reported.

Figure 10: Microstructural meshes considered for the assessment of mesh independency of the method; the meshes have been obtained setting different values of the mesh density parameter $d_{m}$.

Figure 11: Simulated stress-strain curves produced by the four considered meshes for the analyzed 100-grain pseudo-3D polycrystalline specimen; the value of the macroscopic stress component $\Sigma_{y y}$, obtained from averaging the stress tensor over the microstructural volume, is plotted versus the nominal strain defined by $\varepsilon=\Delta \mathrm{U} / 2 H$. 
mentioned, it is the same as those obtained by all the other meshes. As expected, the crack path is normal to the direction of the applied external load. It is worth noting that the failure presents the features of a brittle micro-cracking process and only limited damage is accumulated at the grain interfaces before the failure: almost all the external work is stored in the system as elastic strain energy that is released at the moment of failure.

After the assessment of mesh independency, some other pseudo-3D random specimens subjected to the same load conditions are simulated, to investigate the influence of the microstructure on the micro-cracking process and on the emerging macroscopic stress-strain relationship. The same interface properties as above are assumed. Fig.(12) shows the micro-crack patterns for five different 100-grain microstructures with in-plane grain size ASTM $G=10,\left(\bar{A}_{g r}=126 \mu \mathrm{m}^{2}\right)$ : considering that no pre-existing cracks are included in the simulations, the obtained results, in terms of micro-cracking patterns, are consistent with those reported by Sfantos and Aliabadi [69].

The influence of the number of grains and of the grain size on the micro-cracking is also investigated. Fig.(13) shows the micro-cracking patterns for four 200-grain specimens with grain size ASTM $G=10\left(\bar{A}_{g r}=126 \mu \mathrm{m}^{2}\right)$ and four 200-grain specimens with grain size ASTM $G=12$ $\left(\bar{A}_{g r}=31.5 \mu \mathrm{m}^{2}\right)$. In terms of crack path, the same conclusions as those drawn for the previously analyzed 100-grain specimens can be made. In particular, damage is initiated at interfaces lying on planes normal to the direction of loading and only moderate amount of damage is accumulated at these interfaces before the failure process is initiated. The rupture happens quite suddenly, as it is typical in brittle processes.

Fig.(14) shows the macro stress-strain curves for the analyzed 100-grain and 200-grain specimens. The analyzed ASTM $G=10$ 100-grain and 200-grain microstructures show analogous macroscopic behavior and only moderate softening is observed at the macroscale. A slight difference in the macroscopic stress-strain curves appears between the ASTM $G=10$ and $G=12$ microstructures. In particular, the ASTM $G=12$ specimens, comprised of smaller grains, appear slightly more compliant that the ASTM $G=10$ specimens and show a more pronounced softening behavior close to the critical strain: this is due to the higher surface/volume ratio of the smaller grains, that implies a relatively higher energy dissipation during the damaging process for the smaller grains, and then a less unstable propagation. In other words, during the loading process, each grain stores a certain amount of elastic energy, that is subsequently released and dissipated upon onset of intergranular damage. For smaller grains, a comparatively larger interface per unit volume, with respect to larger grains, and then a more spread damage, can dissipate, during the damaging process, the stored energy, so favoring a less unstable propagation. It is worth stressing, however, that no grain-size effect is implied in terms of crack path in Fig.(13), as all the considered 200-grain specimens differ from each other in terms of morphology and crystallography, so that the crack path differences are only due to the specific features of each specimen.

Finally, Table 4 reports some statistics about the analyzed microstructural meshes, in terms of number of elements, number of interface elements, number of degrees of freedom and measured average time per load increment.

\subsection{Pseudo-3D specimens under compressive load}

In this section, the micro-cracking of some pseudo-3D specimens subjected to unidirectional compressive load is analyzed. The numerical compressive tests are noticeably more demanding than the tensile ones, mainly because of the role played by the frictional contact analysis. 
First, to assess the formulation and its implementation, and to gain some insight into the behavior of simple polycrystals subjected to a compressive load, a microstructure comprised of only $N_{g}=20 \mathrm{SiC}$ grains is considered, Fig.(15). The grains size is ASTM $G=10\left(\bar{A}_{g r}=126 \mu \mathrm{m}^{2}\right)$, while the specimen size is $2 W \times 2 H \times 2 T$ with $H / W=3,4 W H=N_{g} \bar{A}_{g r}$ and $2 T=\sqrt{\bar{A}_{g r}}$. The specimen is subjected to a compressive load, in displacement control, acting along the height $H$ : the rigorous boundary conditions are obtained by inverting the sign of $\Delta \mathrm{U}$ in Eqs.(46). The properties of the cohesive interfaces are specified in Table 5. The parameters $\alpha$ and $\beta$ have been adjusted so to give more weight to the mode II failure, so that in pure mode II the damaging process is activated when $t_{s}=T_{\max } / 2$, see Eq.(17). The effect of the friction on the emerging behavior of the aggregate and on the micro-cracking patterns is investigated, by considering the values $\mu=0.0,0.05,0.1$ and 0.2 for the friction coefficient.

Fig.(16) shows the averaged macroscopic stress-strain curves for the four tested values of friction: it is apparent how the friction influences the load-bearing capability of the specimen, especially after that micro-cracks have formed.

From this simple analysis it is possible to observe that: $a$ ) friction influences, to some extent, the microscopic evolution of micro-cracks; $b$ ) once micro-cracks are formed, the friction affects noticeably the macroscopic post-critical behavior of the aggregate. These simple observations hold their validity also for microstructures comprised of more grains.

After this simple example, four different 100-grain pseudo-3D SiC polycrystals subjected to compression, in displacement control, are considered. The considered grain size is ASTM $G=12\left(\bar{A}_{g r}=31.5 \mu \mathrm{m}^{2}\right)$ and $\mu=0.2$ in all the performed tests. For each microstructure, four different sets of cohesive-frictional parameters are considered, to assess their influence on the microscopic damaging and cracking processes, on the emerging aggregate response and also on the capability of the developed formulation. The assumed sets of interface properties are given in Table 6.

Fig.(17) shows the damage and crack patterns for the last computed load increment of each

\begin{tabular}{cccccc} 
& Microstructure & $N_{\text {els }}$ & $N_{\text {intf }}$ & DoFs & $\bar{T}_{\Delta \lambda}$ \\
\hline \multirow{5}{*}{$N_{g}=100$} & I & 2,786 & 657 & 36,900 & $24 \mathrm{~s}$ \\
& II & 2,924 & 705 & 39,006 & $27 \mathrm{~s}$ \\
& III & 2,521 & 604 & 33,561 & $28 \mathrm{~s}$ \\
& IV & 2,829 & 667 & 37,467 & $32 \mathrm{~s}$ \\
\multirow{2}{*}{$N_{g}=200$} & V & 2,898 & 670 & 38,142 & $24 \mathrm{~s}$ \\
ASTM $G=10$ & I & 5,240 & 1,233 & 69,534 & $65 \mathrm{~s}$ \\
& II & 5,376 & 1,324 & 72,216 & $69 \mathrm{~s}$ \\
& III & 6,097 & 1,470 & 81,333 & $129 \mathrm{~s}$ \\
\multirow{2}{*}{$N_{g}=200$} & IV & 5,703 & 1,372 & 76,023 & $420 \mathrm{~s}$ \\
ASTM $G=12$ & I & 5,347 & 1,283 & 71,217 & $180 \mathrm{~s}$ \\
& II & 6,001 & 1,476 & 80,577 & $202 \mathrm{~s}$ \\
& III & 6,221 & 1,550 & 83,889 & $197 \mathrm{~s}$ \\
& IV & 6,019 & 1,546 & 81,999 & $307 \mathrm{~s}$ \\
\hline
\end{tabular}

Table 4: Some statistics about the analyzed pseudo-3D microstructures. $N_{\text {els }}$ - number of elements; $N_{\text {intf }}$ - number of interfaces; DoFs - number of degrees of freedom; $\bar{T}_{\Delta \lambda}$ - average time per load increment. Tests performed on single 12-core nodes of the $c x 1$ HPC system at Imperial College London, with 24 GBs of required virtual memory. 
Figure 12: Micro-damage and -cracking patterns for five different 100-grain random specimens with in-plane grain size ASTM $G=10$ subjected to the same tensile load as in Fig.(9) (please refer to the electronic version of the paper for colour interpretation).

Figure 13: Micro-cracking patterns for the simulated 200-grain specimens subjected to tensile load. (a): grain size ASTM $G=10 ;(b):$ ASTM $G=12$. The strain levels $\varepsilon$ of the last computed increment are indicated for each specimen. Please refer to the electronic version of the paper for colour interpretation.

Figure 14: Macroscopic stress-strain curves for the 100- and 200-grain pseudo-3D microstructures shown in Figs.(12-13) and subjected to tensile load. In each sub-plot the curves corresponding to the analyzed microstructures are reported from left to right, so that the leftmost curve in the left plot corresponds to the 100-grain specimen marked as I in Fig.(12) and so on. The relevant volume average stress component is plotted versus the corresponding nominal strain, as in Fig.(11).

Figure 15: Micro-cracking patterns for different values of the friction coefficient $\mu$ for the 20-grain SiC microstructure, ASTM $G=10$, subjected to compressive load. The microscopic crack patterns are shown in for the selected values of nominal applied strain $\varepsilon=0.2 \%, 0.25 \%$ and at the last simulated strain for each value of friction.

Figure 16: Stress-strain curves for different values of the friction coefficient $\mu$ for the 20 -grain $\mathrm{SiC}$ microstructure shown in Fig.(15) and subjected a compressive load acting along the specimen longer side. The relevant volume average stress component is plotted against the corresponding nominal strain. 


\begin{tabular}{ccccccc}
$T_{\max }[\mathrm{MPa}]$ & $\alpha$ & $\beta$ & $G_{I I} / G_{I}$ & $\mu$ & $\bar{d}_{E q .(36)}$ & $\xi$ \\
\hline 500 & $1 / 4$ & $1 / 2$ & 1 & $\{0,0.05,0.1,0.2\}$ & 0.8 & 0 \\
\hline
\end{tabular}

Table 5: Sets of cohesive-frictional parameters for the 20-grain pseudo-3D micro-specimens subjected to compressive load.

considered microstructure: each column corresponds to a microstructure while each row corresponds to a set of interface parameters. The corresponding macroscopic stress-strain curves for each microstructure and set of parameters are plotted in Fig.(18). It is observed that the damage patterns match with those reported by Sfantos and Aliabadi for the purely two-dimensional case [69].

To conclude these comments, it is worth noting how the frictional-cohesive terms influence the microstructural failure patterns and then the emerging aggregate behavior: this can be inferred from the comparison between the third and fourth rows in Fig.(17) and the corresponding stressstrain curves in (18). These two sets of analysis differ only for the value of the parameter $\bar{d}$ appearing in Eq.(36), that accounts for a higher or lower energy dissipation due to friction, during the decohesion. On the other hand, the pre-critical behavior seems to depend mainly on the value of the cohesive strength $T_{\max }$, as it can be inferred from the effect of the interface settings B.

\subsection{D specimens under tensile load}

After tuning the method with the pseudo-three-dimensional analysis, the behavior of some fully three-dimensional microstructures subjected to a unidirectional tensile load has been investigated. Four different specimens, comprised of $N_{g}=200$ fully three-dimensional SiC grains, have been tested. The grain size is ASTM $G=12$ (calculated number of grains per $\mathrm{mm}^{3}$ : $\left.n / v=4,527 \cdot 10^{6}[104]\right)$. The specimens' size is $2 W \times 2 W \times 2 H$ with $H / W=2$, their volume is $V=8 H W^{2}=N_{g} \bar{V}_{g r}$, where $\bar{V}_{g r}$ is the estimated average grain volume. The mesh density is specified by $d_{m}=0.5$, that already fulfills the requirements given in Section 3.4. The properties of the interfaces are uniform and they are given in Table 7.

The specimens were subjected to a tensile uniform displacement applied over the end bases and acting along the height direction, as it is shown in Fig.(21), while the lateral sides were not constrained (zero tractions acting on their surface).

Fig.(19) shows the three-dimensional micro-cracking evolution for the four analyzed specimens. It is apparent how, also in the fully three-dimensional case, the damage is accumulated on interfaces mainly normal to direction of the external applied load.

\begin{tabular}{cccccc} 
Settings & $T_{\max }[\mathrm{MPa}]$ & $\alpha$ & $\beta$ & $G_{I I} / G_{I}$ & $\bar{d}_{E q .(36)}$ \\
\hline $\mathrm{A}$ & 500 & $1 / 2$ & 1 & $1 / 2$ & 0.2 \\
$\mathrm{~B}$ & 250 & $1 / 2$ & 1 & $1 / 2$ & 0.2 \\
$\mathrm{C}$ & 500 & $1 / 8$ & $1 / 4$ & 2 & 0.2 \\
$\mathrm{D}$ & 500 & $1 / 8$ & $1 / 4$ & 2 & 0.8 \\
\hline
\end{tabular}

Table 6: Sets of cohesive-frictional parameters considered in the analysis of the 100-grain pseudo-3D micro-specimens subjected to compressive loads. 
However, it is observed that the three-dimensional geometry renders the aggregates much more compliant than their pseudo-3D counterparts. This is evident from the analysis of the macroscopic stress-strain curves reported in Fig.(20), that show marked softening for all the analyzed cases (it is worth noting that the properties of the interfaces have been kept the same as those of the pseudo-3D tests).

Finally, the amplified deformed configuration of another three-dimensional specimen under tensile load, with the same statistical features as the previous ones, is shown in Fig.(21).

Table 8 gives some information about the size of the microstructural meshes and their analysis time.

\subsection{D specimens under compressive load}

To conclude, the compressive behavior of four random 200-grain SiC fully three-dimensional specimens, with grain size ASTM $G=12$, has been investigated. The same microstructures already considered for the tensile tests have been analyzed. For these tests, the interface properties are given by in Table 9. Also here $\alpha$ and $\beta$ have been chosen so to give more weight to the mode II failure.

The macroscopic stress-strain curves for the considered specimens are given in Fig.(22) while Fig.(23) highlights the intergranular damage at the last computed value of strain. It is apparent that, in the case of compressive load, the damage is comparatively more widespread than in the case of tensile load. The first interfaces that fail usually are inclined with respect to the direction of loading, as already observed in the analogous pseudo-3D cases.

An amplified plot of the deformed-cracked configuration for the specimens II and IV, at the last computed value of nominal strain, is given in Fig.(24).

\section{Discussion and further developments}

In this work a new computational model for the analysis of intergranular damage and failure in three-dimensional polycrystalline aggregates has been developed. The performed numerical

\begin{tabular}{ccccccc}
$T_{\max }[\mathrm{MPa}]$ & $\alpha$ & $\beta$ & $G_{I I} / G_{I}$ & $\mu$ & $\bar{d}_{E q .(36)}$ & $\xi$ \\
\hline 500 & 1 & $\sqrt{2}$ & 1 & 0.05 & 0.8 & 0
\end{tabular}

Table 7: Sets of cohesive-frictional parameters for the 3D micro-specimens subjected to tensile load.

\begin{tabular}{ccccc} 
Specimen & $N_{\text {elements }}$ & $N_{\text {interfaces }}$ & DoFs & $\bar{T}_{\Delta \lambda}$ \\
\hline I & 17,031 & 7,709 & 222,660 & $5,130 \mathrm{~s}$ \\
II & 16,525 & 7,582 & 285,201 & $7,434 \mathrm{~s}$ \\
III & 16,834 & 7,652 & 289,242 & $5,599 \mathrm{~s}$ \\
IV & 16,666 & 7,587 & 286,560 & $4,551 \mathrm{~s}$ \\
\hline
\end{tabular}

Table 8: Some statistics about the analyzed 3D microstructures subjected to tensile load. $N_{\text {els }}$ - number of elements; $N_{\text {intf }}$ - number of interfaces; DoFs - number of degrees of freedom; $\bar{T}_{\Delta \lambda}$ - average time per load increment. Tests performed on single 12-core nodes of the cxl HPC system at Imperial College London, with 48 GBs of required virtual memory. 
Figure 17: Micro-damaging and micro-cracking patterns for different values of the cohesive-frictional parameters for four random 100-grain pseudo-3D polycrystals subjected to compressive load (please refer to the electronic version of the paper for colour interpretation). The values of the nominal strain $\varepsilon$ applied at the last computed increment are indicated.

Figure 18: Macroscopic stress-strain curves for the four random 100-grain specimens subjected to compression, for the considered different values of the cohesive-frictional parameters. The volume average stress component $\Sigma_{y y}$ is plotted against the nominal applied strain.

Figure 19: Micro-cracking patterns for four different random 200-grain (ASTM $G=12$ ) 3D microstructures subjected to tensile load. The values of the applied nominal strain $\varepsilon$ are indicated. Each interface is assigned an RGB colour proportional to its own damage level, and a transparency inversely proportional to the damage level itself; in this way, the more an interface is damaged, the more it is visible, avoiding that heavily damaged or even failed interfaces are hidden behind barely damaged blue interfaces. Please refer to the electronic version of the paper for colour interpretation.

Figure 20: Macroscopic stress-strain curves for the four considered random 200-grain ASTM $G=12$ three-dimensional microstructures subjected to tensile load. The volume average stress component $\Sigma_{z z}$ is plotted against the nominal applied strain.

Figure 21: Amplified plot of a stretched-cracked random 200-grain 3D specimen under tensile load. The frame is captured upon failure and shows, from four different viewpoints, the propagation of a 3D crack, mainly normal to the direction of the external load.

Figure 22: Macroscopic stress-strain curves for the four considered random ASTM $G=12$ 200-grain three-dimensional microstructures subjected to compressive load. The volume average stress component $\Sigma_{z z}$ is plotted against the nominal applied strain $\varepsilon$

Figure 23: Micro-cracking patterns at the last computed value of applied nominal strain $\varepsilon$ for the four different random 200-grain three-dimensional microstructures subjected to compressive load. The same graphical strategy as that used for the previous three-dimensional tensile tests has been used. Please refer to the electronic version of the paper for colour interpretation.

Figure 24: Amplified plot of the two compressed-cracked random 3D specimens II and IV at the last computed value of nominal strain $\varepsilon$ indicated in Fig.(23). Views of the four sides are shown. 


\begin{tabular}{ccccccc}
$T_{\max }[\mathrm{MPa}]$ & $\alpha$ & $\beta$ & $G_{I I} / G_{I}$ & $\mu$ & $\bar{d}_{E q .(36)}$ & $\xi$ \\
\hline 500 & $1 / 8$ & $1 / 4$ & 2 & 0.2 & 0.8 & 0 \\
\hline
\end{tabular}

Table 9: Sets of cohesive-frictional parameters for the 3D micro-specimens subjected to compressive load.

tests have shown the potential of the proposed method. However, some aspects deserve further consideration and some directions of further investigation may be discussed.

First of all, it should be noted that the present formulation is restricted to intergranular failure: transgranular failure is not taken into account. This aspect is likely to be responsible for some interlocking observed in the compressive tests. However, from the purely numerical point of view, the presence of transgranular cracking could be taken into account in the presented framework by using either a multi-region boundary element approach or a dual boundary element formulation to model the existence of new transgranular crack surfaces [68]. The main difficulty in this context would be the definition of physically based criteria to address the intergranular-transgranular competition mechanism in a fully three-dimensional picture.

Regarding the aggregate morphology, although the use of Voronoi tessellations offers the modelling simplification of flat grain boundaries, the formulation is not restrained to this simple case and it could be readily extended to the analysis of microstructures with more complex grain morphology, for example those generated through experimental reconstruction. Grain morphologies like those considered by Simonovski and Cizelj $[48,49]$, obtained by X-ray diffraction contrast tomography, could be modeled straightforwardly using linear or quadratic continuous triangular or quadrangular boundary elements [68].

Anisotropic elasticity has been adopted as constitutive model for the grains. However, as mentioned in the Introduction, crystal plasticity might be considered in some applications, see for example [49]. According to the crystal plasticity theory, in single crystals the plastic deformation occurs through simple shear on some specific slip planes. In general, in the framework of Boundary Elements, plastic deformation can be accommodated by using either an initial strain or stress approach [68]. However, this implies the introduction, in the boundary integral equations, of some volume terms and then the need of a volume mesh. At the state of the art, however, it is not clear to the authors if this would offer, in the present context, some advantage over more common finite element formulations.

On the other hand, the presence of intergranular corrosion, can be readily included in the model. According to [49], the cohesive properties of susceptible grain boundaries can be degraded to model the action of some corrosive environment. These effects can be considered in the formulation and the only concern is that the degradation should be introduced so to not upset the numerical stability.

Given its nature, the model seems to be particularly suitable and very promising for the investigation of all the aspects related to the tailoring of the macroscopic material properties through the modification of intergranular interface properties, in the sense Grain boundary engineering. From this point of view, the use of cohesive models, in the context of the developed boundary integral formulation, appears to provide a general framework, potentially able to include, with some ease, other chemical-physical effects (e.g. the presence of segregates) in the modelling of the crystal boundaries. In this context it is however crucial to assess the predictive capability of the model through suitable experimental validation, especially now that the three-dimensional microstructural characterization of materials is becoming technically and economically more af- 
fordable. It is equally important to develop suitable procedures for the measurement and or estimation of the microstructural parameters involved in the model, e.g. the cohesive strength or the critical displacement jumps.

The capability of modelling the nucleation and coalescence of cracks at the crystal level is an important aspect for the multiscale analysis of materials. The nucleation of cracks at the macroscopic component level is usually modelled by using semi-empirical relationships. The availability of a microstructural model allows one to get rid of the assumptions at the macro-scale, as the cracks are initiated at the micro-scale and then transferred to the macro-scale when damage has spread beyond a certain threshold. The conceptual development and numerical implementation of such aspects, in the framework of a multiscale modelling approach to material degradation and fracture of polycrystalline materials $[35,37,38]$, will be the focus of further studies.

It is worth mentioning that, in a multiscale context, the proposed formulation can be used as a microscale model to estimate material degradation or plastic deformation in a macroscale FE model, according to what has been proposed in [35]. On the other hand, the intergranular crack model developed here can certainly be used in a FEM, see again [49], although the introduction of cohesive elements implies additional degrees of freedom, on the contrary of what happens here, which precisely constitutes an advantage of the method.

In conclusion, some numerical/computational aspects deserve further study. Referring to the compressive tests, see for example Fig.(18), it is worth observing that, to take the analysis beyond the critical points, it might be necessary to implement some numerical scheme to improve the convergence of the analysis in the post-critical branch $[105,106]$, like for example generalpurpose arc-length procedures $[107,108]$ or some additional dissipative terms in the cohesive laws [105].

Moreover, performing the numerical tests, it has been noticed that the memory required by the solver PARDISO, see Appendix C, during the symbolic and numerical factorization phases, constitutes a bottleneck of the numerical method. From this point of view, the development of specific iterative Krylov solvers, for sparse systems having the structure of system (11), would be a remarkable achievement $[109,110]$. Moreover, being the analysis of polycrystalline microstructures a large-scale problem, the consideration of special techniques, with the aim of enhancing both the memory and time performance of the developed model, would be an interesting subject: an example in this context could be given by the use of Hierarchical Matrices in conjunction with iterative solvers [111, 112].

\section{Conclusions}

A new three-dimensional formulation for the analysis of intergranular degradation and failure in polycrystalline materials has been developed. The polycrystalline microstructures have been represented as three-dimensional Voronoi tessellations, able to retain the main morphological and crystallographic features of polycrystalline aggregates. The micromechanical model is expressed in terms of intergranular fields, namely displacement jumps and tractions. The nucleation and evolution of intergranular damage has been followed using an extrinsic irreversible cohesive law at the intergranular interfaces: this resulted particularly straightforward, being the formulation itself expressed in terms of grain boundary variables. It has been shown how the used tractionseparation laws ensure the energetic path dependency of the decohesion process and are able to deal with mixed-mode failures. Upon complete intergranular failure a frictional contact analysis has been introduced to model the intergranular micro-cracking process, taking into account 
separation, contact and sliding between the micro-crack surfaces. A new cohesive-frictional law has been used for smoothing the transition from the cohesive to the frictional regime, when the failure happens in mode II under compressive load.

Several numerical tests, both on pseudo- and fully-three-dimensional polycrystalline aggregates, have demonstrated the capability of the formulation to model the nucleation, evolution, coalescence of multiple damage and cracks. The influence of several intergranular parameters (cohesive strength, fracture toughness, friction) on the microcracking patterns and on the macroscopic response of the polycrystals has been demonstrated. It has been shown how the full three-dimensionality of the grains can affect the macroscopic response of the aggregate, making it relevantly different from the response of pseudo-3D aggregates with the same interface properties. The reported results have shown a good agreement with data available in the literature. Overall, the performed tests have demonstrated the potentiality of the technique for the study of the changes induced in macroscopic material properties by changes in the microstructural material features. For its nature, the developed formulation appears particularly promising in the framework of grain boundary engineering.

The analysis of degradation and failure in three-dimensional polycrystalline microstructures remains a remarkable computational task. However, due to the advantage given by the need of modelling only the grain boundaries, the computational effort for the developed formulation remains relatively low.

\section{Acknowledgements}

This research was supported by a Marie Curie Intra European Fellowship within the 7th European Community Framework Programme (Project No 274161).

\section{Appendix A. Anisotropic Fundamental solutions}

The 3D fundamental solutions $U_{k p}(\boldsymbol{x}, \boldsymbol{y})$ in Eq.(4) are obtained solving

$$
\Gamma_{i k}\left(\nabla_{\boldsymbol{x}}\right) U_{k p}(\boldsymbol{x}, \boldsymbol{y})=-\delta_{i p} \delta(\boldsymbol{x}-\boldsymbol{y})
$$

where $\Gamma_{i k}\left(\nabla_{x}\right)=c_{i j k l} \partial x_{j} \partial x_{l}, \delta_{i p}$ is the Kronecker delta and $\delta(\boldsymbol{x}-\boldsymbol{y})$ is the Dirac delta. In general $U_{i j}$ can be expressed as

$$
U_{i j}=\frac{1}{8 \pi^{2} r} \int_{0}^{2 \pi} \Gamma_{i j}^{-1}(\phi) d \phi
$$

where $r=\|\boldsymbol{x}-\boldsymbol{y}\|, \Gamma(\phi)=\Gamma_{i j}[\boldsymbol{z}(\phi)]=c_{i k j l} z_{k}(\phi) z_{l}(\phi)$ and $\boldsymbol{z}(\phi)$ is the generic unit vector lying on a plane normal to the direction defined by the unit vector $\boldsymbol{b}=(\boldsymbol{y}-\boldsymbol{x}) /\|(\boldsymbol{y}-\boldsymbol{x})\|$, expressed in terms of the angle $\phi$ formed with a chosen reference direction on the same plane.

The traction Green's functions $T_{i j}$ can be conveniently expressed as

$$
T_{i j}=c_{k j m p} \frac{\partial U_{m i}}{\partial x_{p}} n_{k}
$$

where $n_{k}$ are the components of the unit vector identifying the plane over which the tractions act. The derivation and effective use of the anisotropic Green's functions for the BEM has been the subject of several investigations (see [90, 112] and references therein for further details). 


\section{Appendix B. Grain boundary element discretization}

The presented formulation only requires meshing of the grain surfaces. The adopted discretization strategy is detailed in [70]; however it is briefly reviewed here, to introduce the parameter $d_{m}$ recalled in the numerical experiments.

Each grain is represented as a convex polyhedron bounded by convex polygonal flat faces. These are discretized into plane linear triangular elements, while linear discontinuous triangular elements are used for representing the unknown grain boundary fields. The actual mesh is created using the mesh generator Triangle (http://www. cs.cmu.edu/ quake/triangle.html, [113]), that provides 2D high-quality meshes of plane surfaces.

Since grain edges and faces present relevant scatter in terms of length and area, it is important to ensure a suitable level of mesh homogeneity: to this end, a discretization parameter $d_{m}$ is introduced, so that the generic edge of length $L_{e}$ is split into a number of segments

$$
n_{s}=\operatorname{round}\left(d_{m} \frac{L_{e}}{\bar{L}_{e}}\right)
$$

where $\bar{L}_{e}$ denotes the average edge length and $\operatorname{round}(x)$ is the function returning the value of the argument rounded to the integer closer to $x$ itself (excluding zero). The parameter $d_{m}$ defines the number of segments in which the average-length cell edge is split, and it is then a measure of the mesh density. After edge splitting, the segment vertices are used as seeds for the face triangulation. The procedure leads to the creation of satisfactorily homogeneous meshes.

\section{Appendix C. System solution implementation}

Regarding the numerical system solution, it is important to realize that the coefficient matrix in Eq.(11) is highly sparse: for such reason, the use of specialized techniques is desirable, with the aim of accelerating the solution with respect to general linear solvers. In this work the system solution has been tackled by using the solver PARDISO (http: //www . pardiso-project . org/; $[114,115])$, which is included in the Intel ${ }^{\circledR}$ Math Kernel Library. PARDISO is a general parallel solver for the direct solution of unsymmetric and symmetric sparse linear systems on shared memory multiprocessors. In the present study, it has been used both as an in-core solver, when few grains have been considered, and as an out-of-core solver, when an higher number of grains has been analyzed. The out-of-core option is usually slower than the in-core one, due to the need of writing on and reading from disk.

PARDISO has been used to address the full incremental-iterative solution of the material degradation process. As described in Section 4.1, during the microstructural evolution, some matrix elements, initially corresponding to the coefficients of the interface compatibility equations, gradually change, according to the degradation stages of the interface itself and, accordingly, system (12) has to be solved several times. To accelerate the whole process, PARDISO has been used combining both direct and iterative solution methods. This is achieved:

i) by retaining the same sparsity pattern for the evolving system matrix, throughout the entire solution process, so to compute the symbolic factorization only once at the beginning of the analysis and use it in all the subsequent solution steps; 
ii) by using the numerical LU factorization of the system matrix corresponding to a certain state of the microstructure and directly computed by PARDISO for preconditioning the Krylowsubspace iterative solution of a certain number of subsequent steps.

The last point is based on the observation that, if the changes in the system matrix are small, the previously computed LU factorization is close to the factorization of the new system matrix, so that it can be used for preconditioning the iterative solution of the new system itself. If the iteration convergence is not reached, the solver automatically switches back to the direct numerical factorization. This mixed direct-iterative solution strategy is switched on by suitably setting the input parameter iparm (4) of PARDISO. The interested reader is referred to the PARDISO documentation for further details.

\section{References}

[1] Z. Hashin, Analysis of Composite Materials - A survey, ASME Journal of Applied Mechanics 50 (1983) 481-505.

[2] T. Mura, Micromechanics of defects in solids, Mechanics of Elastic and Inelastic Solids, Kluwer Academic Publishers, Dordrecht, The Netherlands, second revised edition edition, 1987.

[3] S. Nemat-Nasser, M. Hori, Micromechanics: overall properties of heterogeneous materials, North-Holland, Elsevier, Amsterdam, The Netherlands, second revised edition edition, 1999.

[4] B. L. Adams, T. Olson, The mesostructure - properties linkage in polycrystals, Progress in Materials Science 43 (1998) 1-88.

[5] A. G. Crocker, P. E. J. Flewitt, G. E. Smith, Computational modelling of fracture in polycrystalline materials, International Materials Reviews 50 (2005) 99-124.

[6] P. Shewmon, Grain boundary cracking, Metallurgical and Materials Transactions A 29 (1998) $1535-1544$.

[7] M. P. Seah, Segregation and the strength of grain boundaries, Proceedings of the Royal Society of London. A. Mathematical and Physical Sciences 349 (1976) 535-554.

[8] C. L. Briant, S. K. Banerji, Intergranular failure in steel: the role of grain-boundary composition, International Metals Reviews 23 (1978) 164-199.

[9] P. E. J. Flewitt, R. K. Wild, Grain Boundaries: Their Microstructure and Chemistry, Wiley-VCH, 2001.

[10] D. Lee, E. Barrera, J. Stark, H. Marcus, The influence of alloying elements on impurity induced grain boundary embrittlement, Metallurgical and Materials Transactions A 15 (1984) 1415-1430.

[11] Y. K. Weng, C. J. McMahon, Interaction of phosphorus, carbon, manganese, and chromium in intergranular embrittlement of iron, Materials Science and Technology 3 (1987) 207-216.

[12] C. Liu, K. Abiko, M. Tanino, Role of chromium in the intergranular fracture of high purity Fe-P-Cr alloys with small amounts of carbon, Materials Science and Engineering: A 176 (1994) 363 - 369.

[13] W. T. Geng, A. J. Freeman, R. Wu, G. B. Olson, Effect of Mo and Pd on the grain-boundary cohesion of Fe, Phys. Rev. B 62 (2000) 6208-6214.

[14] W. T. Geng, A. J. Freeman, G. B. Olson, Influence of alloying additions on the impurity induced grain boundary embrittlement, Solid State Communications 119 (2001) 585 - 590.

[15] S. P. Lynch, B. C. Muddle, T. Pasang, Mechanisms of brittle intergranular fracture in Al-Li alloys and comparison with other alloys, Philosophical Magazine A 82 (2002) 3361-3373.

[16] D.-D. Shen, S.-H. Song, Z.-X. Yuan, L.-Q. Weng, Effect of solute grain boundary segregation and hardness on the ductile-to-brittle transition for a Cr-Mo low-alloy steel, Materials Science and Engineering: A 394 (2005) 53 -59 .

[17] P. Lejcek, Grain Boundary Segregation in Metals, volume 136 of Springer Series in Materials Science, Springer Berlin Heidelberg, 2010.

[18] M. Yamaguchi, J. Kameda, K.-I. Ebihara, M. Itakura, H. Kaburaki, Mobile effect of hydrogen on intergranular decohesion of iron: first-principles calculations, Philosophical Magazine 92 (2012) 1349-1368.

[19] E. P. George, C. T. Liu, H. Lin, D. P. Pope, Environmental embrittlement and other causes of brittle grain boundary fracture in $\mathrm{Ni}_{3} \mathrm{Al}$, Materials Science and Engineering: A 192-193, Part 1 (1995) 277 - 288.

[20] W. Wei, H. J. Grabke, The effect of alloying elements on the grain boundary segregation of phosphorus in iron and the intergranular corrosion of the Fe-P system, Corrosion Science 26 (1986) 223 - 236.

[21] C. Leas, E. D. Hondros, Intergranular microchemistry and stress corrosion cracking, Proceedings of the Royal Society of London. A. Mathematical and Physical Sciences 377 (1981) 477-501. 
[22] C. J. McMahon Jr, Hydrogen-induced intergranular fracture of steels, Engineering Fracture Mechanics 68 (2001) $773-788$.

[23] U. Krupp, Dynamic embrittlement-time-dependent quasi-brittle intergranular fracture at high temperatures, International Materials Reviews 50 (2005) 83-97.

[24] T. Watanabe, S. Tsurekawa, The control of brittleness and development of desirable mechanical properties in polycrystalline systems by grain boundary engineering, Acta Materialia 47 (1999) $4171-4185$.

[25] K. M. Döbrich, C. Rau, C. E. Krill III, Quantitative characterization of the three-dimensional microstructure of polycrystalline Al-Sn using X-ray microtomography, Metallurgical and Materials Transactions A 35A (2004) 1953-1961.

[26] C. Zhang, A. Suzuki, T. Ishimaru, M. Enomoto, Characterization of Three-dimensional grain structure in polycrystalline iron by serial sectioning, Metallurgical and Materials Transactions A 35A (2004) 1927-1933.

[27] M. A. Groeber, B. K. Haley, M. D. Uchic, D. M. Dimiduk, S. Gosh, 3D reconstruction and characterization of polycrystalline microstructures using a FIB-SEM system, Materials Characterization 57 (2006) 259-273.

[28] A. King, G. Johnson, D. Engelberg, W. Ludwig, J. Marrow, Observations of intergranular stress corrosion cracking in a grain-mapped polycrystal, Science 321 (2008) 382-385.

[29] M. Groeber, S. Ghosh, M. D. Uchic, D. M. Dimiduk, A framework for automated analysis and simulation of 3D polycrystalline microstructures. Part 1: Statistical characterization, Acta Materialia 56 (2008) 1257-1273.

[30] W. Ludwig, A. King, P. Reischig, M. Herbig, E. M. Lauridsen, S. Schmidt, H. Proudhon, S. Forest, P. Cloetens, S. R. du Roscoat, J. Y. Buffire, T. J. Marrow, H. F. Poulsen, New opportunities for 3d materials science of polycrystalline materials at the micrometre lengthscale by combined use of $\mathrm{x}$-ray diffraction and $\mathrm{x}$-ray imaging, Materials Science and Engineering: A 524 (2009) 69 - 76

[31] S. Rahimi, D. L. Engelberg, J. A. Duff, T. J. Marrow, In situ observation of intergranular crack nucleation in a grain boundary controlled austenitic stainless steel, Journal of Microscopy 233 (2009) 423-431.

[32] M. Herbig, A. King, P. Reischig, H. Proudhon, E. M. Lauridsen, J. Marrow, J.-Y. Buffire, W. Ludwig, 3-d growth of a short fatigue crack within a polycrystalline microstructure studied using combined diffraction and phasecontrast x-ray tomography, Acta Materialia 59 (2011) 590 - 601

[33] A. King, W. Ludwig, M. Herbig, J.-Y. Buffire, A. A. Khan, N. Stevens, T. J. Marrow, Three-dimensional in situ observations of short fatigue crack growth in magnesium, Acta Materialia 59 (2011) $6761-6771$

[34] P. E. J. Flewitt, R. Moskovic, Contribution of multiscale materials modelling for underwriting nuclear pressure vessel integrity, Materials Science and Technology 20 (2004) 553-566.

[35] G. K. Sfantos, M. H. Aliabadi, Multi-scale boundary element modelling of material degradation and fracture, Computer Methods in Applied Mechanics and Engineering 196 (2007) 1310-1329.

[36] E. Askari, F. Bobaru, R. B. Lehoucq, M. L. Parks, S. A. Silling, O. Weckner, Peridynamics for multiscale materials modeling, Journal of Physics: Conference Series 125 (2008) 012078.

[37] S. K. Sfantos, M. H. Aliabadi, Multiscale modeling of structural failure process due to material degradation and mixed mode cracking, Journal of Multiscale Modelling 01 (2009) 57-77.

[38] V. P. Nguyen, M. Stroeven, L. J. Sluys, Multiscale continuous and discontinuous modeling of heterogeneous materials: a review on recent developments, Journal of Multiscale Modelling 03 (2011) 229-270.

[39] M. Kamaya, Influence of grain boundaries on short crack growth behaviour of IGSCC, Fatigue \& Fracture of Engineering Materials \& Structures 27 (2004) 513-521.

[40] N. Sukumar, D. J. Srolovitz, T. J. Baker, J. H. Prévost, Brittle fracture in polycrystalline microstructures with the extended finite element method, International Journal for Numerical Methods in Engineering 56 (2003) 20152037

[41] S. Das, A. Shterenlikht, I. C. Howard, E. J. Palmiere, A general method for coupling microstructural response with structural performance, Proceedings of the Royal Society A: Mathematical, Physical and Engineering Science 462 (2006) 2085-2096

[42] A. Rinaldi, D. Krajcinovic, P. Peralta, Y.-C. Lai, Lattice models of polycrystalline microstructures: A quantitative approach, Mechanics of Materials 40 (2008) $17-36$.

[43] G. Smith, A. Crocker, G. Hughes, P. Flewitt, Three-dimensional modelling of fracture in polycrystals, in: E. E. Gdoutos (Ed.), Fracture of Nano and Engineering Materials and Structures, Springer Netherlands, 2006, pp. 823824.

[44] T. J. Marrow, L. Babout, A. P. Jivkov, P. Wood, D. Engelberg, N. Stevens, P. J. Withers, R. C. Newman, Three dimensional observations and modelling of intergranular stress corrosion cracking in austenitic stainless steel, Journal of Nuclear Materials 352 (2006) 62-74.

[45] G. M. Hughes, G. E. Smith, P. Flewitt, A. G. Crocker, The brittle fracture of polycrystalline zinc, Proceedings of the Royal Society A: Mathematical, Physical and Engineering Science 463 (2007) 2129-2151.

[46] D. Catoor, K. S. Kumar, Crack-grain boundary interactions in zinc bicrystals, Philosophical Magazine 91 (2011) 2154-2185.

[47] I. Simonovski, L. Cizelj, Automatic parallel generation of finite element meshes for complex spatial structures, 
Computational Materials Science 50 (2011) 1606 - 1618

[48] I. Simonovski, L. Cizelj, Computational multiscale modeling of intergranular cracking, Journal of Nuclear Materials 414 (2011) 243 - 250

[49] I. Simonovski, L. Cizelj, Towards modeling intergranular stress corrosion cracks on grain size scales, Nuclear Engineering and Design 246 (2012) 107 - 114.

[50] J. A. R. Bomidi, N. Weinzapfel, F. Sadeghi, Three-dimensional modelling of intergranular fatigue failure of fine grain polycrystalline metallic mems devices, Fatigue \& Fracture of Engineering Materials \& Structures 35 (2012) 1007-1021.

[51] Y. Toi, T. Kiyosue, Damage mechanics models for brittle microcracking solids based on three-dimensional mesoscopic simulations, Engineering Fracture Mechanics 50 (1995) 11 - 27.

[52] M. Kamaya, M. Itakura, Simulation for intergranular stress corrosion cracking based on a three-dimensional polycrystalline model, Engineering Fracture Mechanics 76 (2009) 386 - 401

[53] A. Musienko, G. Cailletaud, Simulation of inter- and transgranular crack propagation in polycrystalline aggregates due to stress corrosion cracking, Acta Materialia 57 (2009) 3840 - 3855.

[54] N. Chandra, H. Li, C. Shet, H. Ghonem, Some issues in the application of cohesive zone models for metal-ceramic interfaces, International Journal of Solids \& Structures 39 (2002) 2827-2855.

[55] C. Shet, N. Chandra, Analysis of energy balance when using cohesive zone models to simulate fracture processes, Journal of Engineering Materials and Technology, Transactions of the ASME 124 (2002) 440-450.

[56] V. Tvergaard, Effect of fibre debonding in a whisker-reinforced metal, Materials Science and Engineering: A 125 (1990) $203-213$

[57] X.-P. Xu, A. Needleman, Void nucleation by inclusion debonding in a crystal matrix, Modelling and Simulation in Materials Science and Engineering 1 (1993) 111-132.

[58] G. T. Camacho, M. Ortiz, Computational modelling of impact damage in brittles materials, International Journal of Solids \& Structures 33 (1996) 2899-2938.

[59] M. Ortiz, A. Pandolfi, Finite-deformation irreversible cohesive elements for three-dimensional crack-propagation analysis, International Journal for Numerical Methods in Engineering 44 (1999) 1267-1282.

[60] J. Zhai, M. Zhou, Finite element analysis of micromechanical failure modes in a heterogeneous ceramic material system, International Journal of Fracture 101 (2000) 161-180. 10.1023/A:1007545105723.

[61] J. Zhai, V. Tomar, M. Zhou, Micromechanical simulation of dynamic fracture using the cohesive finite element method, Journal of Engineering Materials and Technology - Tra 126 (2004) 179-191.

[62] H. D. Espinosa, P. D. Zavattieri, A grain level model for the study of failure initiation and evolution in polycrystalline brittle materials. Part I: Theory and numerical implementation, Mechanics of Materials 35 (2003) 333-364.

[63] H. D. Espinosa, P. D. Zavattieri, A grain level model for the study of failure initiation and evolution in polycrystalline brittle materials. Part II: numerical examples, Mechanics of Materials 35 (2003) 365-394.

[64] Y. J. Wei, L. Anand, Grain-boundary sliding and separation in polycrystalline metals: application to nanocrystalline fcc metals, Journal of the Mechanics and Physics of Solids 52 (2004) 2587-2616.

[65] S. Maiti, K. Rangaswamy, P. H. Geubelle, Mesoscale analysis of dynamic fragmentation of ceramics under tension, Acta Materialia 53 (2005) $823-834$.

[66] T. Zhou, C. Huang, H. Liu, J. Wang, B. Zou, H. Zhu, Crack propagation simulation in microstructure of ceramic tool materials, Computational Materials Science 54 (2012) 150 - 156.

[67] L. C. Wrobel, M. H. Aliabadi, The boundary element method: applications in thermo-fluids and acoustics., volume 1, John Wiley \& Sons Ltd, England, 2002.

[68] M. H. Aliabadi, The boundary element method: applications in solids and structures., volume 2, John Wiley \& Sons Ltd, England, 2002.

[69] G. K. Sfantos, M. H. Aliabadi, A boundary cohesive grain element formulation for modelling intergranular microfracture in polycrystalline brittle materials, International Journal for Numerical Methods in Engineering 69 (2007) 1590-1626.

[70] I. Benedetti, M. H. Aliabadi, A three-dimensional grain boundary formulation for microstructural modelling of polycrystalline materials, Computational Materials Science 67 (2013) 249-260.

[71] E. Ferrié, J.-Y. Buffire, L. Wolfgang, A. Gravouil, L. Edwards, Fatigue crack propagation: In situ visualization using X-ray microtomography and 3D simulation using the extended finite element method, Acta Materialia 54 (2006) 1111-1122.

[72] D. J. Rowenhorst, A. C. Lewis, G. Spanos, Three-dimensional analysis of grain topology and interface curvature in a $\beta$-titanium alloy, Acta Materialia 58 (2010) 5511-5519.

[73] M. Groeber, S. Ghosh, M. D. Uchic, D. M. Dimiduk, A framework for automated analysis and simulation of 3D polycrystalline microstructures. Part 2: Synthetic structure generation, Acta Materialia 56 (2008) 1274-1287.

[74] Y. Bhandary, S. Sarkar, M. Groeber, M. D. Uchic, D. M. Dimiduk, S. Gosh, 3D polycrystalline microstructure reconstruction from FIB generated serial sections for FE analysis, Computational Materials Science 41 (2007) 
222-235.

[75] A. Musienko, A. Tatschl, K. Schmidegg, O. Kolednik, R. Pippan, G. Cailletaud, Three-dimensional finite element simulation of a polycrystalline copper specimen, Acta Materialia 55 (2007) 4121-4136.

[76] T. I. Zohdi, P. Wriggers, An introduction to computational micromechanics, volume 20 of Lecture Notes in Applied and Computational Mechanics, Springer, Berlin, 2005

[77] S. Kumar, S. K. Kurtz, J. R. Banavar, M. G. Sharma, Properties of a three-dimensional Poisson-Voronoi tessellation: a Monte Carlo study, Journal of Statistical Physics 67 (1992) 523-551.

[78] S. Kumar, S. K. Kurtz, Monte-Carlo study of angular and edge length distributions in a three-dimensional PoissonVoronoi tessellation, Materials Characterization 34 (1995) 15-27.

[79] M. Tanemura, Statistical distributuions of Poisson Voronoi cells in two and three dimensions, Forma 18 (2003) 221-247.

[80] R. Quey, P. R. Dawson, F. Barbe, Large scale 3D random polycrystals for the finite element method: Generation, meshing and remeshing, Computer Methods in Applied Mechanics and Engineering 200 (2011) 1729-1745.

[81] F. Fritzen, T. Böhlke, E. Schnack, Periodic three-dimensional mesh generation for crystalline aggregates based on Voronoi tessellations, Computational Mechanics 43 (2009) 701-713.

[82] S. Kumar, S. K. Kurtz, Simulation of material microstructure using a 3D Voronoi tessellation: Calculation of effective thermal expansion coefficient of polycrystalline materials, Acta Metallica 42 (1994) 3917-3927.

[83] S. Kumar, S. K. Kurtz, V. K. Agarwala, Micro-stress distribution within polycrystalline aggregate, Acta Mechanica 114 (1996) 203-216.

[84] M. Nygårds, P. Gudmundson, Three-dimensional periodic Voronoi grain models and micromechanical FEsimulations of a two phase steel, Computational Materials Science 24 (2002) 513-519.

[85] F. Barbe, L. Decker, D. Jeulin, G. Cailletaud, Intergranular and intragranular behavior of polycrystalline aggregates. Part 1: F.E. model, International Journal of Plasticity 17 (2001) 513-536.

[86] F. Barbe, S. Forest, G. Cailletaud, Intergranular and intragranular behavior of polycrystalline aggregates. Part 2 : Results, International Journal of Plasticity 17 (2001) 537-563.

[87] C. H. Rycroft, Voro++: A three-dimensional Voronoi cell library in C++, Chaos 19 (2009) 041111.

[88] R. B. Wilson, T. A. Cruse, Efficient implementation of anisotropic three dimensional boundary-integral equation stress analysis, International Journal for Numerical Methods in Engineering 12 (1978) 1383-1397.

[89] M. M. Mehrabadi, S. C. Cowin, Eigentensors of linear anisotropic elastic materials, Quarterly Journal of Mechanics and Applied Mathematics 43 (1990) 15-41.

[90] N. A. Schclar, Anisotropic analysis using boundary elements, volume 20 of Topics in Engineering, Computational Mechanics Publications, 1994.

[91] C. Y. Wang, M. Denda, 3D BEM for general anisotropic elasticity, International Journal of Solids \& Structures 44 (2007) 7073-7091.

[92] M. J. van den Bosch, P. J. G. Schreurs, M. G. D. Geers, An improved description of the exponential xu and needleman cohesive zone law for mixed-mode decohesion, Engineering Fracture Mechanics 73 (2006) 1220 1234.

[93] L. Snozzi, J.-F. Molinari, A cohesive element model for mixed mode loading with frictional contact capability, International Journal for Numerical Methods in Engineering (2013) 510-526.

[94] K. W. Man, M. H. Aliabadi, D. P. Rooke, Bem frictional contact analysis: Load incremental technique, Computers \& Structures 47 (1993) 893 - 905.

[95] K. W. Man, M. H. Aliabadi, D. P. Rooke, Bem frictional contact analysis: Modelling considerations, Engineering Analysis with Boundary Elements 11 (1993) 77 - 85.

[96] J. A. Garrido, A. Foces, F. París, An incremental procedure for three-dimensional contact problems with friction, Computers \& Structures 50 (1994) $201-215$.

[97] V. Tomar, J. Zhai, M. Zhou, Bounds for element size in a variable stiffness cohesive finite element model, International Journal for Numerical Methods in Engineering 61 (2004) 1894-1920.

[98] J. R. Rice, Mathematical analysis in the mechanics of fracture, in: H. Liebowitz (Ed.), Fracture: An Advanced Treatise, Vol 2 of Mathematical Fundamentals, Academic Press, N.Y., 1968.

[99] I. Benedetti, M. H. Aliabadi, A grain boundary formulation for the analysis of three-dimensional polycrystalline microstructures, Key Engineering Materials 525-526 (2013) 1-4.

[100] M. Ostoja-Starzewski, Material spatial randomness: from statistical to representative volume element, Probabilistic Engineering Mechanics 21 (2006) 112-132.

[101] G. Arlt, G. R. Schodder, Some elastic constants of silicon carbide, Journal of the Acoustical Society of America 37 (1965) 384-386.

[102] W. R. L. Lambrecht, B. Segall, M. Methfessel, M. van Schilfgaarde, Calculated elastic constants and deformation potentials of cubic SiC, Physical Review B 44 (1991) 3685-3694.

[103] R. D. Carnahan, Elastic properties of silicon carbide, Journal of the American Ceramic Society 51 (1968) 223 224. 
[104] ASTM E112-10, Standard Test Methods for Determining Average Grain Size. (2010). ASTM International. DOI: 10.1520/E0112-10.

[105] Y. F. Gao, A. F. Bower, A simple technique for avoiding convergence problems in finite element simulations of crack nucleation and growth on cohesive interfaces, Modelling and Simulation in Materials Science and Engineering 12 (2004) 453.

[106] N. Hu, Y. Zemba, H. Fukunaga, H. H. Wang, A. M. Elmarakbi, Stable numerical simulations of propagations of complex damages in composite structures under transverse loads, Composites Science and Technology 67 (2007) $752-765$.

[107] M. A. Crisfield, A fast incremental/iterative solution procedure that handles snap-through, Computers \& Structures 13 (1981) 55-62.

[108] V. Mallardo, C. Alessandri, Arc-length procedures with bem in physically nonlinear problems, Engineering Analysis with Boundary Elements 28 (2004) 547 - 559.

[109] F. C. de Araújo, E. F. d'Azevedo, L. J. Gray, Boundary-element parallel-computing algorithm for the microstructural analysis of general composites, Computers \& Structures 88 (2010) 773-784.

[110] F. C. de Araújo, E. F. d'Azevedo, L. J. Gray, Constructing efficient substructure-based preconditioners for BEM systems of equations, Engineering Analysis with Boundary Elements 35 (2011) 517-526.

[111] I. Benedetti, M. H. Aliabadi, G. Davì, A fast 3D dual boundary element method based on hierarchical matrices, International Journal of Solids \& Structures 45 (2008) 2355-2376.

[112] I. Benedetti, A. Milazzo, M. H. Aliabadi, A fast dual boundary element method for 3D anisotropic crack problems, International Journal for Numerical Methods in Engineering 80 (2009) 1356-1378.

[113] J. R. Shewchuk, Triangle: Engineering a 2D Quality Mesh Generator and Delaunay Triangulator, in: M. Lin, D. Manocha (Eds.), Applied Computational Geometry: Towards Geometric Engineering, volume 1148 of Lecture Notes in Computer Science, Springer-Verlag, Berlin, 1996, pp. 203-222. (From the First ACM Workshop on Applied Computational Geometry.).

[114] O. Schenk, K. Gärtner, Solving Unsymmetric Sparse Systems of Linear Equations with PARDISO, Journal of Future Generation Computer Systems 20 (2004) 475-487.

[115] O. Schenk, K. Gärtner, On fast factorization pivoting methods for symmetric indefinite systems, Elec. Trans. Numer. Anal. 23 (2006) 158-179. 